\title{
Improved Interference-Free Channel Allocation in Coordinated Multiuser Multi-Antenna Open-Access Small Cells
}

\author{
Redha M. Radaydeh, Senior Member, IEEE, Ammar Zafar, Member, IEEE, Fawaz S. Al-Qahtani, Member, IEEE, \\ and Mohamed-Slim Alouini, Fellow, IEEE
}

\begin{abstract}
This paper investigates low-complexity joint interference avoidance and desired link improvement for single channel allocation in multiuser multi-antenna access points (APs) for open-access small cells. It is considered that an active user is equipped with an atenna array that can be used to suppress interference sources but not to provide spatial diversity. On the other hand, the operation of APs can be coordinated to meet design requirements, and each of which can unconditionally utilize assigned physical channels. Moreover, each AP is equipped with uncorrelated antennas that can be reused simultaneously to serve many active users. The analysis provides new approaches to exploit physical channels, transmit antennas, and APs to mitigate interference, while providing the best possible link gain to an active user through the most suitable interference-free channel. The event of concurrent service requests placed by active users on a specific interference-free channel is discussed for either interference avoidance through identifying unshared channels or desired link improvement via multiuser scheduling. The applicability of the approaches to balance downlink loads is explained, and practical scenarios due to imperfect identification of interference-free channels and/or scheduled user are thoroughly investigated. The developed results are applicable for any statistical and geometric models of the allocated channel to an active user as well as channel conditions of interference users. They can be used to study various performance measures. Numerical and simulation results are presented to explain some outcomes of this work.
\end{abstract}

Index Terms-small cells, open-access cells, resource allocation, interference avoidance, desired link improvement, reconfigurable antenna systems, multiuser systems, multiuser scheduling, load balancing, coordinated transmission, antenna array steering, statistical modeling, interference-free channel identification.

\section{INTRODUCTION}

Copyright (c) 2015 IEEE. Personal use of this material is permitted. However, permission to use this material for any other purposes must be obtained from the IEEE by sending a request to pubs-permissions@iee.org.

Manuscript received XXXX; accepted XXXX. The editor coordinating the review of this paper and approving it for publication was XXXX.

R. M. Radaydeh is with the Electrical Engineering Program, College of Engineering, Alfaisal University, Riyadh, Saudi Arabia (email: rradaydeh@alfaisal.edu).

A. Zafar is with Centre for Real-Time Information Networks, University of Technology Sydney, Sydney, Australia (e-mail: ammar.zafar@uts.edu.au).

F. Al-Qahtani is with Electrical \& Computer Engineering Program, Texas A\&M University at Qatar, Doha, Qatar (e-mail: fawaz.alqahtani@qatar.tamu.edu).

M.-S. Alouini is with Computer, Electrical and Mathematical Sciences \& Engineering (CEMSE) Division, King Abdullah University of Science and Technology (KAUST), Thuwal, Makkah Province, Saudi Arabia (e-mail: slim.alouini@kaust.edu.sa).

The work of F. Al-Qahtani was supported by JSREP grant No. 3-039-2-010 from the Qatar National Research Fund (a member of Qatar Foundation). The statements made herein are solely the responsibility of the authors.
$\mathbf{T}$ HE expanded use of wireless services has resulted in emerging technologies to meet the increasing demand on applications over extended spaces. One of such technologies is to employ overlaid access points (APs) within the existing cellular infrastructure to enhance coverage when cellular services are limited. For instance, small-cell APs, such as in femtocell technology [1], [2], use the fixed broadband technology as backhaul to users' traffic. Such small-cell APs are expected to operate at relatively low power, be simple to install, and provide adequate performance [1].

The use of multiple short-coverage APs in existing macrocellular network creates many management and technical issues, such as radio resources distribution, service coverage and coordination, power consumption, quality of service satisfaction, and operational costs. Among the techniques that can be adopted to reduce the impact of these issues are multiple-antenna configurations [3]-[5], power control [6]-[9], handoff schemes [10], cooperative communication [11]-[16], adaptive resource management and interference mitigation schemes [17]-[19], and radio sensing algorithms [20], [21]. However, an important factor that is always of practical demand is to maintain the design and operational complexity as low as possible, while maximizing resource sharing. This paper addresses a desirable scenario wherein resources are accessible anytime by any deployed small-cell AP without prior distribution.

With open resource sharing, the service management to active users and the effect of interference from various origins can limit the achieved system capacity. The level of interference experienced specifically on downlink by each active user is also affected by the access control strategy in small-cell APs [22]-[24]. Particularly, while the closed-access scheme allows only authorized users to use an AP resources, the openaccess scheme is suitable to serve cellular users in public areas. The latter scheme can reduce the macrocell interference that may be experienced by nearby femtocell users. This reduction is due to the fact that a femtocell AP can serve an active macrocell user that exists inside that femtocell coverage space, thereby avoiding a potential high-power macrocell interference. However, the open-access scheme results in increasing handoff rate, increasing co-tier interference, and limiting resources available to small-cell users. The methodology herein considers the open-access scheme, and it aims to develop quantitative measures for interference-free resources from an active user perspective. The channel identification is linked to 
the capacity of available resources as well as to the interference suppression capability of each active user.

The adopted system model considers that each user is equipped with an array of highly-correlated antennas (i.e., spacing between antenna elements is of order of half wavelength). On the other hand, an AP is equipped of uncorrelated reconfigurable antennas (i.e., antennas are sufficiently separated by multiple of the wavelength). Moreover, each user can receive downlink service from any of the deployed APs, and can access only one of the available physical channel from a specific antenna in a deployed AP. Although the focus is on single interference-free channel assignment scenario, the analysis herein can be modified to study other scenarios, such as multiple interference-free channel assignment. The latter scenario will be largely based on the developed methodologies for interference-free channel identification, and it involves interesting case studies that will be treated in future work.

The physical channel and/or transmit antenna per each AP can be reused to reduce the likelihood of service interruption of active users. As a result, concurrent active users who access the same channel simultaneously are assigned different codes (e.g., through code division multiple access (CDMA)). To support this mechanism, the frequency and/or time division multiplexing technique (e.g., orthogonal sub-carriers as in orthogonal frequency division multiplexing (OFDM) and/or time slots as in orthogonal time division multiplexing (OTDM)) has to accommodate with the associated CDMA bandwidth expansion and/or the time division of a packet duration. In this regard, modern wireless communications standards, such as 4G Long-Term Evolution (LTE) Advanced by the 3rd Generation Partnership Project (3GPP), aims to support such hybrid access technologies with the small-cell deployment $[25]^{1}$. However, when there are many active users at the same time, maintaining orthogonality among users becomes infeasible, and hence, interference from different origins is a likely event.

This paper proposes new approaches for downlink channel allocation, which exploits available physical channels, multiple antennas, and multiple APs to mitigate the effect of interference while providing the best possible downlink channel service. The developed approaches relates the best possible channel identification to the availability of interference-free channels per active user. In addition, it exploits collided multiuser requests to either improve the allocated link or avoid interference. The approach adopted for multiuser scheduling capitalizes on the single channel allocation objective at the expense of processing requirements ${ }^{2}$.

The contributions of this paper are summarized as follows.

\footnotetext{
${ }^{1}$ A significant performance improvement in LTE Advanced networks comes from bringing the network closer to users by adding small-cell terminals. However, new techniques for resource allocation, coordinated multi-point operation, and interference management and suppression are of importance, which are of interest in this paper.

${ }^{2}$ Other approaches to extract multi-user diversity in the context of the adopted system model and channel allocation herein can be also developed. Additional treatment of this specific case is beyond the scope of this work.
}

Low-complexity ${ }^{3}$ approaches for joint interference avoidance and improved downlink channel allocation in the context of coordinated multiuser multi-antenna small-cell coverage spaces under open-access strategy are investigated. The system model considers multiple APs to serve as many active users as possible while achieving interference-free downlink channel assignment per served user. Each AP is allocated multiple physical channels and employs multiple reconfigurable antennas to exploit spatial multiplexing. On the other hand, each user receive station is equipped with an array that can not achieve diversity gain, but it can be utilized to mitigate interference sources. Each physical channel and transmit antenna per AP can be reused unconditionally anytime anywhere in the coverage space of interest. The analysis provides new approaches to exploit the availability of physical channels, transmit antennas, and APs in mitigating interference per active user, while improving the user's channel assignment. The collided concurrent requests from active users is also discussed, and treated in the context of interference avoidance or allocated channel improvement. The effect of imperfectness in the channel identification and/or best possible scheduled user are thoroughly investigated for various scenarios. The developed results are applicable for any channel and geometric models, and statistical models of interference sources. The findings can be applied in the context of overlaid wireless technologies over designated coverage spaces based on radio sensing, hybrid multiple-access and multiplexing techniques.

The rest of the paper is organized as follows. Section II presents preliminary discussions on the system model and available resources. Section III discusses the adopted approaches for interference-free channels identification per active user. Section IV obtains the statistics of desired link received power under perfect desired channel identification, and then extends the findings to multiuser access. Section V quantifies the effect of imperfect channel identification and imperfect user identification for several practical cases. Selected numerical and simulation results are discussed in section VI, and conclusions follow in section VII.

\section{Preliminary Discussions}

This section contains two main parts. In the first part, an overview of the adopted system model and available resources is presented. In the second part, the use of available resources and its effect on interference sources are described.

\section{A. Overview of System Model}

The system model herein considers a coverage area that is part of a macrocell in which a number of $K$ small-coverage APs are deployed to serve authorized users. The APs share similar operational properties. Each of which functions according to the open-access control strategy, and it is equipped with a transmit array of $N$ uncorrelated antennas. Note that,

\footnotetext{
${ }^{3}$ The low-complexity is a consequence that the proposed approaches avoid the need for costly or complicated processing techniques, such as advanced optimization and interference alignment. They provide simple strategies for interference avoidance and resource allocation with improved performance and tolerable latency (e.g., refer to subsection III-C for a related discussion).
} 


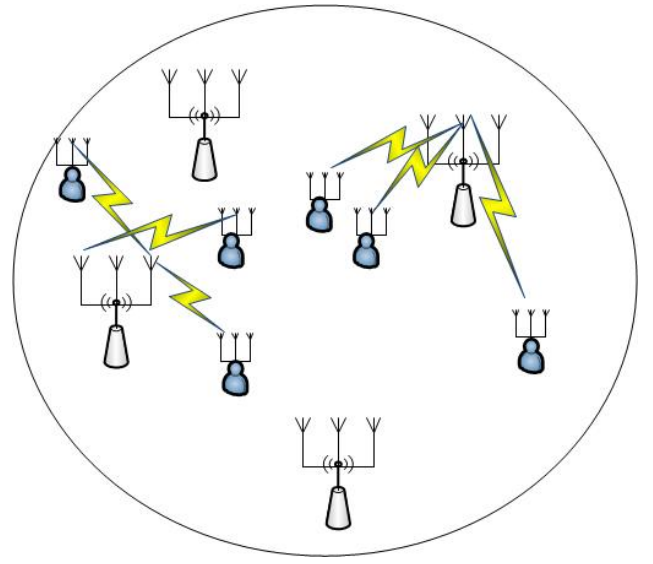

Fig. 1. Adopted system model for single physical channel use. The array antennas at each access point are uncorrelated and separated by using three distinct signatures, and the access points have their unique identification codes. The array antennas at each mobile terminal are highly correlated, and are assumed to be identical for all terminals herein.

since these APs are expected to share similar characteristics as mentioned above, the use of the same array size for all APs is a reasonable assumption. However, the consideration that the deployed APs have different array sizes can be incorporated into the analysis, but it will result in more complicated notations and involved treatment.

The transmit antennas per AP are separated by orthogonal signatures, which can be reassigned to other antennas at a different AP in close vicinity. The use of orthogonal signatures to distinguish among antennas utilizes the spatial domain to generate simultaneous orthogonal channels on the same physical channel, with proper antenna reconfiguration through parallel feedings into antenna devices using a suitable switching circuitry among feed-lines ${ }^{4}$. For instance, the antennas can be arranged to cover specific spaces (see, e.g., [26], [27]). This is particularly useful to meet growing traffic demand at a wireless site, wherein antenna elements may be installed to create sectors, which are distinguished by assigned signatures. The resulting benefit is expected to be an amplification of the capacity by the number of created sectors.

For a user receive station, it is equipped with an array that contains insufficiently spaced antennas due to space restriction. The receive stations may in general have arrays of different sizes, wherein the $j$ th user uses an array of size $L_{j}$ antenna elements, for $j=1,2, \ldots, J_{\max }$, and $J_{\max }$ is the number of authorized users in the coverage area of interest. Fig. 1 shows the adopted system model herein for single physical channel use.

Since the receive antennas at each user are considered to be insufficiently spaced, they can not be useful to provide diversity gain. However, they may be utilized to suppress interference source via steering the array's main lobe electronically to produce nulls (i.e., very low array gains) along the directions of interference sources, while providing pattern's maximum

\footnotetext{
${ }^{4}$ Note that the deployed antenna elements at an AP are treated as a class of pattern reconfigurable antennas [28], which are different from those used for a multiuser beamforming.
}

gain in the direction of the desired source. Therefore, the antenna array at each user will act as a single transmit/receive device but with many degrees of freedom, which are function of the number of deployed antennas, to steer the direction of the array pattern as desired. The steering precision controls the interference suppression capability, and hence, it affects the achieved downlink performance at an active user. The null steering can be implemented through various approaches, including the control of array weights (i.e., amplitude and phase), amplitude-only, or phase-only (see, e.g., [29]-[31], and references therein). For the most complex amplitudephase adjustment, the receive station needs to predict interference sources powers and their associated direction of arrivals (DoAs). And the signatures of interference users can be exploited to improve the prediction accuracy.

Each AP can use a total number of $M$ physical channels (e.g., sub-carriers as in OFDM). These physical channels are known to deployed APs as well as to users receive stations. Therefore, the system is an expansion of that shown in Fig. 1 over disjoint physical layers due to the availability of multiple downlink channels.

\section{B. The Use of Resources and Interference Mitigation}

An active user in the coverage area of interest can be served by one AP through one of its transmit antennas and can be allocated one physical channel at a time, as depicted in Fig. 1. This mechanism is adopted herein in order to reduce the likelihood of users service interruption, decrease the complexity of distributing resources according to users requests, and boost the system sum-capacity by exploiting spatial de-correlation between active users and their variable service requirements (also, refer to section I for a related discussion).

It is considered that the transmit antennas as well as the physical channels that are associated with an AP can be simultaneously reused to serve many active users without any reuse limit. For each AP, the concurrent allocation of the same physical channel through any transmit antenna is expected to increase the level interference. However, the signature separation between transmit antennas results in orthogonal distribution of interference sources on each physical channel. This is expected to improve the accessibility of physical channels in a distributed manner. Although the adopted use of unique identities to distinguish between APs demands higher operating complexity and requires more communications overhead, it is specifically useful to further separate interference sources.

The physical channels, transmit antennas at each AP, and multiple APs, can be further exploited not only to mitigate interference but also to enhance the quality of service to an active user. Moreover, the inherent diversity associated with spatially uncorrelated users can be exploited to further improve the system-level performance via multi-user scheduling along with interference avoidance, although such improvement is expected to affect the deployed APs coverage. 


\section{INTERFERENCE-FREE CHANNEL IDENTIFICATION}

The contents of this section are divided into four parts. In the first part, the events of interference-free and occupied channels from an active user perspective are described. In the second part, the sets of interference-free and occupied channels are characterized. The third part treats the expected time duration to find an interference-free channel. Finally, the fourth part explains the utilization of available channels to balance downlink service loads.

\section{A. Events of Interference-Free and Occupied Channels}

This subsection identifies the events of interference-free and occupied channels. The description is presented for the case when this identification exploits all physical channels, transmit antennas per each AP, and deployed APs (i.e., all available channels) with no restrictions. The developed results in this part are valid for any active user with arbitrary size of its receive array.

1) Access to an Available Channel: Let $J_{\text {act }}$ denotes the number of active users at a time. The term $J_{\text {act }}$ can take on values from $\{0,1,2, \ldots, J\}$, where $J \leq J_{\max }$ refers to the number of users that can concurrently access one of the available physical channels on a transmit antenna from an operating AP. When the probability that the $j$ th user be active is given by $p_{\text {act }, j}=p_{\text {act }}$, for $j=1,2, \ldots, J$ (i.e., users are equally likely to be active anytime anywhere inside the coverage area of interest), it follows that

$$
\operatorname{Pr}\left\{J_{\text {act }}=w\right\}=\left(\begin{array}{c}
J \\
w
\end{array}\right)\left(p_{\text {act }}\right)^{w}\left(1-p_{\text {act }}\right)^{J-w}
$$

which converges to a Poisson distribution with a mean value of $J p_{\text {act }}$ when $J \gg 1$ and $p_{\text {act }} \ll 1$. The value of $J$ counts for all possible users as being interference sources, regardless of their origins.

Define $V_{m, k, n, j}$ as the number of active users that access the $m$ th physical channel on the $n$th transmit antenna from the $k$ th $\mathrm{AP}$ and observed by the $j$ th user. The quantity $V_{m, k, n, j}$ can take on values from the set $\left\{0,1,2, \ldots, J_{\text {act }}\right\}$, where $J_{\text {act }}$ has distribution as given in (1). Moreover, let $p_{m, k, n, i}$ be the probability that the $i$ th active user (i.e., an interference user) accesses the channel of indexes $(m, k, n)$, where $\sum_{n=1}^{M} \sum_{n=1}^{N} \sum_{k=1}^{K} p_{m, k, n, i}=1^{5}$. By utilizing the results in [32], the conditional distribution of $V_{m, k, n, j}$ can be expressed as

$$
\begin{aligned}
& \operatorname{Pr}\left\{V_{m, k, n, j}=v \mid J_{\mathrm{act}}=w\right\} \\
& = \begin{cases}\sum_{\mathcal{A} \in \mathcal{F}_{v}} \prod_{i \in \mathcal{A}} p_{m, k, n, i} \prod_{i^{\prime} \in \mathcal{A}^{c}}\left(1-p_{m, n, k, i^{\prime}}\right), & w \geq v ; \\
0, & w<v,\end{cases}
\end{aligned}
$$

where $\mathcal{F}_{v}$ is the set of all subsets of $v$ integers that can be selected from $\{1,2,3, \ldots, w\}$, and it contains $\left(\begin{array}{l}w \\ v\end{array}\right)$ elements, and $\mathcal{A}^{c}$ is the complement of $\mathcal{A}$. Note that (2) includes the

\footnotetext{
${ }^{5}$ It is worth mentioning herein that the term $p_{m, k, n, i}$ refers to the statistical likelihood that the channel of indexes $(m, n, k)$ is allocated to the $i$ th active user, which is measured as an a priori ratio relative to an expanded record of channel allocation history to that user.
}

fact that $V_{m, k, n, j}$ can not exceed $J_{\text {act }}$ at any time ${ }^{6}$. Using the results in (1) and (2), it can be shown that

$$
\begin{aligned}
& \operatorname{Pr}\left\{V_{m, k, n, j}=v\right\} \\
& =\sum_{w=0}^{J}\left[\sum_{\mathcal{A} \in \mathcal{F}_{v}} \prod_{i \in \mathcal{A}} p_{m, k, n, i} \prod_{i^{\prime} \in \mathcal{A}^{c}}\left(1-p_{m, n, k, i^{\prime}}\right)\right] \operatorname{Pr}\left\{J_{\mathrm{act}}=w\right\} \\
& =\sum_{w=v}^{J}\left[\sum_{\mathcal{A} \in \mathcal{F}_{v}} \prod_{i \in \mathcal{A}} p_{m, k, n, i} \prod_{i^{\prime} \in \mathcal{A}^{c}}\left(1-p_{m, n, k, i^{\prime}}\right)\right] \operatorname{Pr}\left\{J_{\mathrm{act}}=w\right\} .
\end{aligned}
$$

The result in (3) considers all possible events of $J_{\text {act }}$ into the likelihood of occurrence of each $V_{m, k, n, j}$. The expected value of $V_{m, k, n, j}$ can be expressed as $\mathbb{E}\left\{V_{m, k, n, j}\right\}=$ $\sum_{i=1}^{J} p_{\text {act }} p_{m, k, n, i} \leq \mathbb{E}\left\{J_{\text {act }}\right\}$, where the only scenario to have $\mathbb{E}\left\{V_{m, k, n, j}\right\}=\mathbb{E}\left\{J_{\text {act }}\right\}$ is when $p_{m, k, n, i}=1$ for only a specific case of indexes $(m, k, n)$ (i.e., the $i$ th user accesses channel $(m, k, n)$ with probability one) and $p_{m, k, n, i}=0$ for all other remaining channels.

2) Channel Conditions: Capitalizing on the modeling presented in [34], the received interference signals (or users) vector at the $j$ th user receive antenna array during the $l$ th signalling duration, which contains $L_{j}$ entries and referred to as $\mathbf{r}_{\mathrm{I}, m, k, n, j}[l]$, can be expressed, using the superposition principle, as

$$
\begin{aligned}
\mathbf{r}_{\mathrm{I}, m, k, n, j}[l] & =\sum_{i=1}^{v} a_{\mathrm{I}, m, k, n, i}[l] c_{\mathrm{I}, m, k, n, i}[l] \sqrt{E_{\mathrm{I}, m, k, n, i}} \\
& \times z_{\mathrm{I}, m, k, n, i, j} \mathbf{s}_{m, k, n, i, j},
\end{aligned}
$$

where $\mathbf{s}_{m, k, n, i, j}=\left[s_{m, k, n, i, 1, j} s_{m, k, n, j, 2, j} \cdots s_{m, k, n, i, L_{j}, j}\right]^{\mathrm{T}}$ is the steering vector in the direction of signal component associated with the $i$ th interference user, and $a_{\mathrm{I}, m, k, n, i}[l]$, $c_{\mathrm{I}, m, k, n, i}[l] \quad E_{\mathrm{I}, m, k, n, i}$, and $z_{\mathrm{I}, m, k, n, i, j}$ are the information symbol, CDMA code, average transmit energy per symbol, and complex-valued channel coefficient, all associated with $i$ th interference user, respectively.

Note that $z_{\mathrm{I}, m, k, n, i, j}$ is experienced by the $j$ th user due to the downlink service that is provided simultaneously to the $i$ th user on the channel whose indexes are $(m, k, n)$. As the $i$ th user is allocated at the $k$ th AP single transmit antenna (i.e., the $n$th antenna), $z_{\mathrm{I}, m, k, n, i, j}$ is a scalar quantity, but it is observed on the $j$ th user's array of size $L_{j}$. The term $z_{\mathrm{I}, m, k, n, i, j}$ is weighted by $\mathbf{s}_{m, k, n, i, j}$ of size $L_{j}$ since the weight to that user's interference signal can be different from one antenna element to another, according to the null steering algorithm. The description in (4) counts for all possible interference sources on the $j$ th user channel of indexes $(m, k, n)$.

After receive weighting by $\mathbf{w}_{j}=\left[\begin{array}{llll}w_{1, j} & w_{2, j} & \cdots & w_{L_{j}, j}\end{array}\right]^{\mathrm{T}}$ and by the CDMA code of the $j$ th user, the interference power

\footnotetext{
${ }^{6}$ Based on the results in [33], it can be written that $\operatorname{Pr}\left\{V_{m, k, n, j}=v \mid J_{\text {act }}=\right.$ $w\} \sim \frac{e^{-\lambda} \lambda^{v}}{v !}$, where $\lambda=\sum_{i=1}^{w} p_{m, k, n, i}$, which provides a desirable tight result to that in (2) when $w \gg 1$ and $p_{m, k, n, i} \ll 1$.
} 
observed at the $j$ th user's receive station on channel $(m, k, n)$, which is referred to by $s_{\mathrm{I}, m, k, n, j}$, can be written as

$$
\begin{aligned}
s_{\mathrm{I}, m, k, n, j} & =\sum_{i=1}^{v} \zeta_{\mathrm{I}, m, k, n, i, j} E_{\mathrm{I}, m, k, n, i} \\
& \times\left|z_{\mathrm{I}, m, k, n, i, j}\right|^{2}\left|\mathbf{w}_{j}^{\mathrm{H}} \mathbf{s}_{m, k, n, i, j}\right|^{2} .
\end{aligned}
$$

where $\zeta_{\mathrm{I}, m, k, n, i, j} \triangleq \mathbb{E}\left\{c_{\mathrm{I}, m, k, n, i}[l] c_{\mathrm{I}, m, k, n, j}[l]\right\}$, and $\mathbb{E}\{\cdot\}$ is the expectation operator. This term is bounded as $0 \leq$ $\zeta_{\mathrm{I}, m, k, n, i, j} \leq 1$. When the CDMA codes assigned to active users who simultaneously access the same channel $(m, k, n)$ are nonorthogonal, it follows that $\zeta_{\mathrm{I}, m, k, n, i, j}>0$ (i.e., interference can not be eliminated).

For the adopted single channel allocation per active user, the $j$ th user receive station that requests downlink service attempts to identify at least one interference-free channel by searching over all physical channels supported on a transmit antenna from an AP. Herein, null steering algorithm can suppress $L_{j}-1$ resolvable interference users. Therefore, the identification of an interference-free channel is assured as long as at least one of the available physical channels on a transmit antenna from an AP supports a number of resolvable interference users that is below $L_{j}$. Consequently, the receive array weighting $\mathbf{w}_{j}$ needs to satisfy $\mathbf{w}_{j}^{\mathrm{H}} \mathbf{s}_{m, k, n, i, j}=0$, for $i=1,2, \ldots, v$. Then, the likelihood of the event $s_{\mathrm{I}, m, k, n, j} \rightarrow 0$ (i.e., interferencefree channel identification) can be expressed as ${ }^{7}$

$$
\begin{aligned}
& \operatorname{Pr}\left\{s_{\mathrm{I}, m, k, n, j} \rightarrow 0\right\} \\
& =\operatorname{Pr}\left\{V_{m, k, n, j} \leq L_{j}-1\right\} \\
& =\sum_{v=0}^{L_{j}-1} \sum_{w=v}^{J}\left[\sum_{\mathcal{A} \in \mathcal{F}_{v}} \prod_{i \in \mathcal{A}} p_{m, k, n, i} \prod_{i^{\prime} \in \mathcal{A}^{c}}\left(1-p_{m, n, k, i^{\prime}}\right)\right] \\
& \times \operatorname{Pr}\left\{J_{\text {act }}=w\right\} .
\end{aligned}
$$

Moreover, the likelihood of $s_{\mathrm{I}, m, k, n, j} \nrightarrow 0$ (i.e., occupied channel) is characterized as

$$
\begin{aligned}
& \operatorname{Pr}\left\{s_{\mathrm{I}, m, k, n, j} \nrightarrow 0\right\} \\
& =\sum_{v=L_{j}}^{J} \sum_{w=v}^{J}\left[\sum_{\mathcal{A} \in \mathcal{F}_{v}} \prod_{i \in \mathcal{A}} p_{m, k, n, i} \prod_{i^{\prime} \in \mathcal{A}^{c}}\left(1-p_{m, n, k, i^{\prime}}\right)\right] \\
& \times \operatorname{Pr}\left\{J_{\text {act }}=w\right\} .
\end{aligned}
$$

For the case when some interference users have correlated DoAs, their composite component will be treated as a single interference source, regardless of the associated combined power level. For a specific channel $(m, k, n)$, the conditions for interference-free and occupied channels will be specified according to the number of de-correlated DoAs rather than to the actual number of interference users, thereby the effect of a relatively large number of interference users can mitigated.

Note that, for the case of composite interference users are aligned in their DoAs, the orthogonality between physical

\footnotetext{
${ }^{7}$ A relaxed criterion to identify an interference-free channel, which takes into account the effect non-ideal null steering, can be stated as follows: $\operatorname{Pr}\left\{s_{\mathrm{I}, m, k, n, j} \rightarrow^{\epsilon} 0\right\}=\operatorname{Pr}\left\{V_{m, k, n, j} \leq L_{j}-1 \& s_{\mathrm{I}, m, k, n, j}<\epsilon\right\}=$ $\operatorname{Pr}\left\{V_{m, k, n, j} \leq L_{j}-1\right\} \operatorname{Pr}\left\{s_{\mathrm{I}, m, k, n, j}<\epsilon\right\}$, where $\epsilon$ can be set relative to the background noise power floor. The analysis in the remaining parts is valid for this criterion.
}

channels can be useful to establish orthogonal spatial layers. Therefore, the increase in $M$ can potentially lead to a larger number of interference sources that can be suppressed for the same number of steering directions. However, the increase in $M$ involves practical constraints, as will be discussed in the sequel.

Note also that when the DoA of the desired link to the $j$ th user is aligned with that of an interference user, the receive array at the $j$ th user can not be used to suppress such interference, and hence, interference-free channel identification becomes infeasible. In this case, precise transmit antenna configuration is needed separate between desired and undesired spatial links. This has a feasible application in smallcell networks under quasi-static condition, wherein the spatial separation between serving APs and their active users are usually limited.

\section{B. Sets of Interference-Free and Occupied Channels}

Define $s_{\mathrm{I}, j}$ as the interference power that is experienced by the $j$ th user, following the receive interference suppression as discussed in the preceding part. The term $s_{\mathrm{I}, j}$ varies from one channel to another as a result of the statistical behavior of interference powers on different channels. Then, the likelihood that the $j$ th user identifies at least one interference-free channel can be obtained as shown in (8) on the top of the next page.

Define $\mathcal{B}_{j}$ as a set that includes the indexes of interferencefree channels predicted by the $j$ th user, where the $r$ th entry of the set $\mathcal{B}_{j}, r \equiv\left(m^{\prime}, k^{\prime}, n^{\prime}\right)$, for $m^{\prime} \in\left\{1,2, \ldots, M^{\prime} \leq M\right\}$, $k^{\prime} \in\left\{1,2, \ldots, K^{\prime} \leq K\right\}$, and $n^{\prime} \in\left\{1,2, \ldots, N^{\prime} \leq N\right\}$, is equivalent to specific indexes of a predicted interference-free channel. The cardinality of $\mathcal{B}_{j}$, denoted by $\left|\mathcal{B}_{j}\right| \leq M^{\prime} K^{\prime} N^{\prime} \leq$ $M K N$, varies according to the occupancy of the channels. Specifically, the likelihoods of the events $\left|\mathcal{B}_{j}\right| \geq 1$ and $\left|\mathcal{B}_{j}\right|=$ 0 can be written as

$$
\begin{aligned}
& \operatorname{Pr}\left\{\left|\mathcal{B}_{j}\right| \geq 1\right\}=\operatorname{Pr}\left\{s_{\mathrm{I}, j} \rightarrow 0\right\}, \\
& \operatorname{Pr}\left\{\left|\mathcal{B}_{j}\right|=0\right\}=\operatorname{Pr}\left\{s_{\mathrm{I}, j} \nrightarrow 0\right\} .
\end{aligned}
$$

The event that $\left|\mathcal{B}_{j}\right|=0$ is feasible only when the condition $V_{m, k, n, j}>L_{j}-1$ is applicable for all channel indexes $(m, k, n)$. On the other hand, the event that $\left|\mathcal{B}_{j}\right|=1$ occurs when exactly one of the available channels satisfies $V_{m, k, n, j} \leq L_{j}-1$ for a specific $(m, k, n)$, while remaining channels satisfy $V_{m, k, n, j}>L_{j}-1$. Finally, the maximum possible cardinality of $\mathcal{B}_{j}$ (i.e., $\left|\mathcal{B}_{j}\right|=M N K$ ) is feasible when $V_{m, k, n, j} \leq L_{j}-1$ is applicable for all channel indexes $(m, k, n)$.

From the preceding discussion, and for the case when $J \leq$ $L_{j}-1$, it can be written that

$$
\operatorname{Pr}\left\{\left|\mathcal{B}_{j}\right|=b \mid J \leq L_{j}-1\right\}= \begin{cases}0, & b=0,1, \ldots, M N K-1 \\ 1, & b=M N K\end{cases}
$$

which shows that all available channels will be predicted by the $j$ th user as being interference-free as long as the number of interference users (or interference groups of users having independent DoAs) that access any of these channels can be 


$$
\begin{aligned}
\operatorname{Pr}\left\{s_{\mathrm{I}, j} \rightarrow 0\right\} & =1-\prod_{m=1}^{M} \prod_{k=1}^{K} \prod_{n=1}^{N} \operatorname{Pr}\left\{s_{\mathrm{I}, m, k, n, j} \nrightarrow 0\right\} \\
& = \begin{cases}1, & J \leq L_{j}-1 ; \\
1-\prod_{m=1}^{M} \prod_{k=1}^{K} \prod_{n=1}^{N}\left(1-\operatorname{Pr}\left\{V_{m, k, n, j} \leq L_{j}-1\right\}\right), & J>L_{j}-1 .\end{cases}
\end{aligned}
$$

suppressed by the receive array of the $j$ th user. Therefore, the increase in $L_{j}$ can improve the service coverage as it enables the support of an increased number active interference users over the same channel simultaneously, while maintaining the interference-free channel condition at each of supported users. However, this is usually limited by the space constraint at each user receive station as well as the capability of the receive array steering.

Considering now the case when $J>L_{j}-1$, and based on the preceding discussions, it follows that the result in (11) can be obtained, where $\mathcal{Q}_{b}$ is the set of all subsets of $b$ integers, where each of which denotes specific indexes $(m, k, n)$ that can be selected from $\{1,2,3, \ldots, M N K\}$. The set $\mathcal{Q}_{b}$ contains $\left(\begin{array}{c}M N K \\ b\end{array}\right)$ elements. From the results in (11), the undesired situation of $\left|\mathcal{B}_{j}\right|=0$ can be a very rare event with the increase in $M, K$, and/or $N$. However, the increase in $M$ is restricted by the allowed operator resources as well as the availability of hardware and network protocols. Moreover, the increase in $N$ is complicated by the synchronization of transmit multiplexing transmissions to different active users, and it is restricted by the available space to deploy antenna arrays at APs. The increase in $K$ complicates the resource allocation and distribution among active users, as will be discussed below.

\section{Search Period for Interference-Free Channel}

Consider that the operation is managed over successive packet time durations, wherein the traffic conditions and the network parameters remain static over at least a packet time. Each packet time duration contains a guard time period over which the identifications of interference-free channels and the best possible downlink channel (i.e., desired link) are performed sequentially. The length of the guard period is a design parameter that is related to the time needed to predict interference powers non-coherently and their DoAs (to perform receive null steering), and to estimate the desired link power levels on identified interference-free channels (to allocate a suitable interference-free channel to an active user). Moreover, the guard period is influenced by the values of $M$, $K$, and $N$, and the angular steering at each user.

For the specific case when all available channels are found occupied at a given time, the $j$ th user attempts to find at least one interference-free channel by repeating the search over successive packet times (or independent block durations for quasi-static channels). Define $Y_{j}$ as the number of packet times over which available channels occupancies need to be tested in order to identify an interference-free channel by the $j$ th user, and noting that the probability that an interference-free channel will be found is given by $\operatorname{Pr}\left\{\left|\mathcal{B}_{j}\right| \geq 1\right\}$, it follows that the result in (12) on the top of the next page is obtained, where an interference-free channel will be found from the first search trial with probability one when $J \leq L_{j}-1$. The expected value of $Y_{j}$, which is denoted by $\mathbb{E}\left\{Y_{j}\right\}$, can be obtained as

$$
\begin{aligned}
& \mathbb{E}\left\{Y_{j}\right\} \\
& = \begin{cases}1, & J \leq L_{j}-1 ; \\
\frac{1}{1-\prod_{m=1}^{M} \prod_{k=1}^{K} \prod_{n=1}^{N}\left(1-\operatorname{Pr}\left\{V_{m, k, n, j} \leq L_{j}-1\right\}\right)}, & J>L_{j}-1 .\end{cases}
\end{aligned}
$$

From (12) and (13), the increase in $M, N$, and/or $K$ reduces the expected number of packet durations over which the search for an interference-free channel by the $j$ th user will take place. In addition, the increase in $L_{j}$ can decrease the expected value of $Y_{j}$ as it enhances the ability of the $j$ th user receive station to find an interference-free channel very shortly.

\section{Balancing Downlink Loads}

The schemes herein can be viewed as effective approaches to balance downlink service loads among available channels. Specifically, the total number of interference users that the $j$ th user can observe on different channels while still predicting these channels as interference-free is $J_{\max }=M K N J$, where $J \leq L_{j}-1$. Moreover, the concurrent service requests placed by emerging users to access a specific channel can be simultaneously supported as long as the condition $J \leq L_{j}-1$ remains applicable for each user accessing that channel, and hence, multi-user scheduling becomes unnecessary to resolve collided requests.

For the case when $J_{\max }>M K N\left(L_{j}-1\right)$, and when the allocation of available channel to the excess number of active users is made arbitrarily, it follows that the excess number of active users, which is denoted by $\widetilde{J}_{\text {act }}$, can take values from the set $\{0,1,2, \ldots, \widetilde{J}\}$, where $\widetilde{J}=J_{\max }-M K N\left(L_{j}-1\right)$. The results presented in previous parts can be used herein with the terms $J_{\text {act }}, J$, and $V_{m, k, n, j}$ therein are now replaced by $\widetilde{J}_{\text {act }}, \widetilde{J}$, and $\widetilde{V}_{m, k, n, j}=V_{m, k, n, j}-\left(L_{j}-1\right)$, respectively, where $\widetilde{V}_{m, k, n, j}$ denotes the actual number of interference users observed by the $j$ th user on channel $(m, k, n)$. Therefore, the likelihood of observing interference on a specific channel as well as the cardinality of the set $\mathcal{B}_{j}$ become related to the likelihoods of $\widetilde{V}_{m, k, n, j}=0$ and $\widetilde{V}_{m, k, n, j}>0$. However, the aforementioned approaches are not cost-free as they make the best interference-free downlink channel identification to serve an active user a rather conditional process. Specifically, the use of some good interference-free channels in the search for the best possible desired link to an active user may need to be avoided in order to meet such distribution of downlink loads. 


$$
\operatorname{Pr}\left\{\left|\mathcal{B}_{j}\right|=b \mid J>L_{j}-1\right\}=\left\{\begin{array}{l}
\prod_{m=1}^{M} \prod_{k=1}^{K} \prod_{n=1}^{N}\left(1-\operatorname{Pr}\left\{V_{m, k, n, j} \leq L_{j}-1\right\}\right), \quad b=0 ; \\
\prod_{m=1}^{M} \prod_{k=1}^{K} \prod_{n=1}^{N}\left(1-\operatorname{Pr}\left\{V_{m, k, n, j} \leq L_{j}-1\right\}\right) \\
\times \sum_{\mathcal{C} \in \mathcal{Q}_{b}} \prod_{(m, k, n) \in \mathcal{C}}\left(\frac{\operatorname{Pr}\left\{V_{m, k, n, j} \leq L_{j}-1\right\}}{1-\operatorname{Pr}\left\{V_{m, k, n, j} \leq L_{j}-1\right\}}\right), \quad b=1,2, \ldots M N K .
\end{array}\right.
$$

$$
\operatorname{Pr}\left\{Y_{j}=y\right\}= \begin{cases}1, & y=1 \& J \leq L_{j}-1 \\ \left(\operatorname{Pr}\left\{\left|\mathcal{B}_{j}\right|=0\right\}\right)^{y-1} \operatorname{Pr}\left\{\left|\mathcal{B}_{j}\right| \geq 1\right\}, & y=1,2, \ldots \& J>L_{j}-1\end{cases}
$$

\section{Perfect Identification of Desired Channel}

In this section, the perfect identification of the suitable channel to realize the best desired link to an active user is discussed. The case when multiple emerging active users demand an access to the same channel is also investigated from the perspectives of further improving the desired link or specifying unshared interference-free channels per active user.

\section{A. Perfect Simultaneous Channel Identification}

This subsection treats the case when the indexes of the physical channel, transmit antenna, and AP associated with best possible channel per active user are identified simultaneously. This best channel identification is performed over the specified set of interference-free channels by each active user.

For the $j$ th user and when $\left|\mathcal{B}_{j}\right| \geq 1$, let $s_{\mathrm{D},(1), j} \leq s_{\mathrm{D},(2), j} \leq$ $\cdots \leq s_{\mathrm{D},\left(\left|\mathcal{B}_{j}\right|\right), j}$ be the order statistics obtained by arranging $\left\{s_{\mathrm{D}, r, j}\right\}_{r=1}^{\left|\mathcal{B}_{\mathrm{B}}\right|}$ in increasing order of magnitude, where $s_{\mathrm{D}, r, j}$, for $r=1,2, \ldots,\left|\mathcal{B}_{j}\right|$, and $r \equiv(m, k, n)$ as defined above, refers to the desired power observed by the $j$ th on the $r$ th interference-free channel whose indexes are in the set $\mathcal{B}_{j}$. Then, the best possible scenario for single interference-free channel allocation to serve the $j$ th user takes place if

$$
\left.s_{\mathrm{D},\left(\left|\mathcal{B}_{j}\right|\right), j}\right|_{\left|\mathcal{B}_{j}\right| \geq 1}=\max \left\{s_{\mathrm{D}, 1, j}, s_{\mathrm{D}, 2, j}, \ldots, s_{\mathrm{D},\left|\mathcal{B}_{j}\right|, j}\right\} .
$$

Then, the unconditional statistics of the desired power observed by the $j$ th user under perfect identification of the best possible channel for all possible events of the number of interference-free channels, which is denoted by $s_{\mathrm{D}, j, \mathrm{se}}$, can be found as shown in (15) on the top of the next page, where the results in (10) and (11) are incorporated into (15). Note that the event $\left|\mathcal{B}_{j}\right|=0$, which results in $\mathcal{B}_{j}=\varnothing$, where $\varnothing$ denotes the empty set, is excluded.

The results in (14) and (15) treat simultaneous channel identification through arranging all available channels whose indexes belong to $\mathcal{B}_{j}$ according to their desired power strengths, irrespective to their specific indexes. In principle, this approach may be performed at the intended receive station through the use of training sequences to know the states (from the desired power level perspective) of the acceptable set of interference-free channels. However, the corresponding cost is a noticeable increase in processing load and power consumption at the receive station in addition to increased latency. On the other hand, when coordination between APs is feasible, each AP can monitor the channel quality on its transmit antennas and physical channels (e.g., by exploiting channel reciprocity as in TDD scenario). Each active user can then convey the indexes of identified interference-free channels to all operating APs. The APs share their information such that the suitable interference-free channel to serve an active user is identified precisely through identifying the indexes $(m, k, n)$ that belong to the identified set of interference-free channels of that user. Such coordinated APs can distribute the processing load associated with the best possible channel identification for each active user, but it requires overhead between APs as well as from active users.

\section{B. Exploiting Multiuser Access for Desired Channel Improve- ment}

For the case when the identified interference-free channel is found suitable to serve other concurrent active requests, the requests can be scheduled to exploit multiuser gain and to avoid interference amplification on any identified interference-free channel. A possible approach to achieve long-term scheduling fairness in providing downlink service to different users can be realized by rearranging users requests for downlink service in time domain. This shuffling process can be performed through a dedicated control signalling on uplink after normalizing by the associated users' average power levels, thereby avoiding long-term occupancy of a specific channel by a relatively strong user.

Let $\mathcal{D}_{\ell}$ be the set that contains the indexes of active users that identify $\ell$ th channel as being the best downlink channel, where $\ell \equiv(m, k, n)$ is used herein to represent the indexes of a specific channel on which there are collided requests from multiple active users. The cardinality of $\left|\mathcal{D}_{\ell}\right|$ can take on values $\left\{0,1,2, \ldots, J_{0}\right\}$, where $J_{0} \leq J$. Let $s_{\mathrm{D},(1) \text {,se }} \leq$ $s_{\mathrm{D},(2), \text { se }} \leq \cdots \leq s_{\mathrm{D},\left(\left|\mathcal{D}_{\ell}\right|\right) \text {,se }}$ be the order statistics obtained by arranging $\left\{s_{\mathrm{D}, j, \mathrm{se}}\right\}_{j=1}^{\left|\mathcal{D}_{\ell}\right|}$ in increasing order of magnitude. Then, the desired power of the scheduled user can be obtained as

$$
\left.s_{\mathrm{D},\left(\left|\mathcal{D}_{\ell}\right|\right), \mathrm{se}}\right|_{\left|\mathcal{D}_{\ell}\right|>0}=\max \left\{s_{\mathrm{D}, 1, \mathrm{se}}, s_{\mathrm{D}, 2, \mathrm{se}}, \ldots, s_{\mathrm{D},\left|\mathcal{D}_{\ell}\right|, \mathrm{se}}\right\} .
$$

The unconditional statistics of the desired power of the scheduled user under perfect best channel identification by each active user whose index belong to $\mathcal{D}_{\ell}$, which is referred to as $s_{\mathrm{D}, \text { se }}$, can now be written as shown in (17), where

$$
\operatorname{Pr}\left\{\left|\mathcal{D}_{\ell}\right|=v_{0}\right\}=\sum_{\mathcal{S} \in \mathcal{K}_{v_{0}}} \prod_{j \in \mathcal{S}} \tilde{p}_{\ell, j} \prod_{j^{\prime} \in \mathcal{S}^{c}}\left(1-\widetilde{p}_{\ell, j^{\prime}}\right),
$$




$$
\begin{aligned}
\operatorname{Pr}\left\{s_{\mathrm{D}, j, \mathrm{se}}<x\right\} & =\sum_{b=0}^{M N K} \operatorname{Pr}\left\{\left.s_{\mathrm{D},(b), j}\right|_{b \geq 1}<x\right\} \operatorname{Pr}\left\{\left|\mathcal{B}_{j}\right|=b\right\} \\
& = \begin{cases}\prod_{r=1}^{M N K} \operatorname{Pr}\left\{s_{\mathrm{D}, r, j}<x\right\}, & J \leq L_{j}-1 ; \\
\frac{1}{1-\operatorname{Pr}\left\{\left|\mathcal{B}_{j}\right|=0\right\}} \sum_{b=1}^{M N K}\left(\prod_{r=1}^{b} \operatorname{Pr}\left\{s_{\mathrm{D}, r, j}<x\right\}\right) \operatorname{Pr}\left\{\left|\mathcal{B}_{j}\right|=b\right\}, & J>L_{j}-1 .\end{cases}
\end{aligned}
$$

$$
\begin{aligned}
\operatorname{Pr}\left\{s_{\mathrm{D}, \mathrm{se}}<x\right\}= & \sum_{v_{0}=0}^{J_{0}} \operatorname{Pr}\left\{\left.s_{\mathrm{D},\left(v_{0}\right), \mathrm{se}}\right|_{v_{0}>0}<x\right\} \operatorname{Pr}\left\{\left|\mathcal{D}_{\ell}\right|=v_{0}\right\} \\
& =\left\{\begin{array}{l}
\frac{1}{1-\operatorname{Pr}\left\{\left|\mathcal{D}_{\ell}\right|=0\right\}} \sum_{v_{0}=1}^{J_{0}}\left(\prod_{j=1}^{v_{0}} \prod_{r=1}^{M N K} \operatorname{Pr}\left\{s_{\mathrm{D}, r, j}<x\right\}\right) \operatorname{Pr}\left\{\left|\mathcal{D}_{\ell}\right|=v_{0}\right\}, \quad J \leq L_{j}-1 ; \\
\frac{1}{1-\operatorname{Pr}\left\{\left|\mathcal{D}_{\ell}\right|=0\right\}} \sum_{v_{0}=1}^{J_{0}}\left(\prod_{j=1}^{v_{0}} \frac{1}{1-\operatorname{Pr}\left\{\left|\mathcal{B}_{j}\right|=0\right\}}\right. \\
\left.\times \sum_{b=1}^{M N K}\left(\prod_{r=1}^{b} \operatorname{Pr}\left\{s_{\mathrm{D}, r, j}<x\right\}\right) \operatorname{Pr}\left\{\left|\mathcal{B}_{j}\right|=b\right\}\right) \operatorname{Pr}\left\{\left|\mathcal{D}_{\ell}\right|=v_{0}\right\}, \quad J>L_{j}-1 .
\end{array}\right.
\end{aligned}
$$

$$
\begin{aligned}
\widetilde{p}_{\ell, j} & =\operatorname{Pr}\left\{j \text { th user accesses the } \ell \text { th channel }\left.\& s_{\mathrm{I}, \ell, j} \rightarrow 0 \& s_{\mathrm{D}, \ell, j}\right|_{\left|\mathcal{B}_{j}\right| \geq 1}=\max _{r=1, \ldots,\left|\mathcal{B}_{j}\right|, r \neq \ell}\left\{s_{\mathrm{D}, 1, j}, s_{\mathrm{D}, 2, j}, \ldots, s_{\mathrm{D},\left|\mathcal{B}_{j}\right|, j}\right\}|| \mathcal{B}_{j} \mid\right\} \\
& =p_{\ell, j}\left(\sum_{v=0}^{L_{j}-1} \sum_{w=v}^{J}\left[\sum_{\mathcal{A} \in \mathcal{F}_{v}} \prod_{i \in \mathcal{A}} p_{\ell, i} \prod_{i^{\prime} \in \mathcal{A}^{c}}\left(1-p_{\ell, i^{\prime}}\right)\right] \operatorname{Pr}\left\{J_{\mathrm{act}}=w\right\}\right) \\
& \times\left(\frac{1}{1-\operatorname{Pr}\left\{\left|\mathcal{B}_{j}\right|=0\right\}} \sum_{b=1}^{M N K}\left(\operatorname{Pr}\left\{s_{\mathrm{D}, \ell, j}=\max _{r=1, \ldots, b, r \neq \ell}\left\{s_{\mathrm{D}, 1, j}, s_{\mathrm{D}, 2, j}, \ldots, s_{\mathrm{D}, b, j}\right\}\right\}\right) \operatorname{Pr}\left\{\left|\mathcal{B}_{j}\right|=b\right\}\right)
\end{aligned}
$$

and $\mathcal{K}_{v_{0}}$ is the set of all subsets of $v_{0}$ integers that can be selected from $\left\{1,2,3, \ldots, J_{0}\right\}$ and it contains $\left(\begin{array}{l}J_{0} \\ v_{0}\end{array}\right)$ elements, and $\mathcal{S}^{c}$ is the complement of $\mathcal{S}$. The term $\widetilde{p}_{\ell, j}$ refers to the probability that the $\ell$ th channel is accessible of the $j$ th user, it is found to be interference-free, and it is found to be the best possible downlink channel to serve that user. Therefore, it is given in (19), where

$$
\begin{aligned}
& \operatorname{Pr}\left\{s_{\mathrm{D}, \ell, j}=\max _{r=1, \ldots, b, r \neq \ell}\left\{s_{\mathrm{D}, 1, j}, s_{\mathrm{D}, 2, j}, \ldots, s_{\mathrm{D}, b, j}\right\}\right\} \\
& =\int_{0}^{\infty}\left[\prod_{r=1, r \neq \ell}^{b} \operatorname{Pr}\left\{s_{\mathrm{D}, r, j}<x\right\}\right] f_{s_{\mathrm{D}, \ell, j}}(x) d x,
\end{aligned}
$$

and $f_{s_{\mathrm{D}, \ell, j}}(x)$ represents the distribution of $s_{\mathrm{D}, \ell, j}$.

The scheme adopted herein for exploiting multi-user concurrent requests is implemented sequentially following the identification of interference-free channels for active users. It is only needed when there are collided access requests placed by emerging active users on a common interferencefree channel.

\section{Exploiting Multiuser Access for Unshared Interference-free Channels Identification}

The channel allocation process from the desired link improvement perspective can be rather managed by distributing users' requests on their predicted interference-fee channels such that collided requests for a service on any interferencefree channel are resolved. Specifically, the set of identified interference-free channels by an active user can be examined against other sets of concurrent emerging users. Then those channels that are unshared in other sets are considered a suitable subset for interference-free and collided-free channels, which can be then exploited to improve the desired link performance. Define $\widetilde{\mathcal{B}}_{j}$ as the set of interference-free channel that are identified by the $j$ th user but not by any other concurrently active user. It can be then written that

$$
\widetilde{\mathcal{B}}_{j}=\mathcal{B}_{j} \backslash \bigcup_{q=1, q \neq j}^{J} \mathcal{B}_{q}, j=1,2, \ldots, J
$$

When $\left|\widetilde{\mathcal{B}}_{j}\right|>0$, it follows that the best possible channel without collision from other concurrent active users can be identified from the set $\widetilde{\mathcal{B}}_{j}$, and hence, multi-user scheduling is avoided. The cardinality of $\widetilde{\mathcal{B}}_{j}$ can take on values from the set of $\{0,1, \ldots, M N K\}$.

The probability that a specific channel of indexes $(m, k, n)$ is found interference-free by the $j$ th user and unshared by any 
other active user can be expressed as

$$
\begin{aligned}
& \operatorname{Pr}\left\{s_{\mathrm{I}, m, k, n, j} \rightarrow 0 \& \text { unshared }\right\} \\
& =\operatorname{Pr}\left\{V_{m, k, n, j} \leq L_{j}-1\right\} \prod_{q=1, q \neq j}^{J} \operatorname{Pr}\left\{V_{m, k, n, q}>L_{q}-1\right\} .
\end{aligned}
$$

The increase in the number of active concurrent users will complicate the search process for unshared channels and increase the processing load. However, this search guarantees no additional multi-user scheduling.

The likelihoods of the two extreme cases of $\left|\widetilde{\mathcal{B}}_{j}\right| \geq 1$ and $\left|\widetilde{\mathcal{B}}_{j}\right|=0$ are expressed as

$$
\begin{aligned}
\operatorname{Pr}\left\{\left|\widetilde{\mathcal{B}}_{j}\right| \geq 1\right\} & =1-\operatorname{Pr}\left\{\left|\widetilde{\mathcal{B}}_{j}\right|=0\right\} \\
\operatorname{Pr}\left\{\left|\widetilde{\mathcal{B}}_{j}\right|=0\right\} & =\prod_{m=1}^{M} \prod_{k=1}^{K} \prod_{n=1}^{N}\left(1-\operatorname{Pr}\left\{V_{m, k, n, j} \leq L_{j}-1\right\}\right. \\
& \left.\times \prod_{q=1, k \neq j}^{J} \operatorname{Pr}\left\{V_{m, k, n, q}>L_{q}-1\right\}\right)
\end{aligned}
$$

From the preceding discussion, and for the case when $J \leq$ $L_{j}-1$ and $J>L_{q}-1$, for $q \neq j$, it can be written that

$$
\begin{aligned}
& \operatorname{Pr}\left\{\left|\widetilde{\mathcal{B}}_{j}\right|=b \mid J \leq L_{j}-1 \& J>L_{q}-1, q \neq j\right\} \\
& = \begin{cases}0, & b=0,1, \ldots, M N K-1 ; \\
1, & b=M N K .\end{cases}
\end{aligned}
$$

And for the case when $J>L_{j}-1$, for $j=1,2, \ldots, J$, it gives the result shown in (25) on the top of the next page, where $\mathcal{G}_{b}$ is the set of all subsets of $b$ integers, where each of which denotes specific indexes $(m, k, n)$ that can be selected from $\{1,2,3, \ldots, M N K\}$.

The unconditional statistics of the desired power associated with identified best possible channel that is drawn from the set $\widetilde{\mathcal{B}}_{j}$ will have similar form to that given in (15) but with the likelihoods of $\left|\widetilde{\mathcal{B}}_{j}\right|$ presented in (24) and (25) replace those of $\left|\mathcal{B}_{j}\right|$ therein. Note that, for the specific case when $\left|\widetilde{\mathcal{B}}_{j}\right|=0$, multiuser scheduling can not be avoided, hence, the treatment of this specific case can be conducted in a much similar procedure to that described in subsection IV-B. However, and from (23), it is seen that this event becomes very rare with the increase in $M, K, N$, and/or $L_{j}$, but its occurrence increases with $J$ and/or the sizes of arrays at other interference users; namely $L_{q}$, for $q \neq j$.

The preceding joint identification of interference-free and unshared downlink channels can reduce the active user's performance gain due to the decrease in the cardinality of the set of interference-free channels (discussed in the preceding parts) as well as the absence of multiuser scheduling gain. However, it can improve system-level performance through reducing the likelihood of service interruption of active users who are not selected during the multiuser scheduling process, thereby enhancing the overall service coverage.

\section{IMPERFECT DESIRED CHANNEL IDENTIFICATION}

This section addresses the effect of imperfect desired channel identification. This imperfectness can be due to inaccurate statistical prediction and/or statistical ordering of desired power levels on predicated interference-free channels per active user. It is observed when an error takes place in one or more channel indexes, which are from $r \equiv\left(m^{\prime}, k^{\prime}, n^{\prime}\right) \in \mathcal{B}_{j}$. The analysis herein covers the cases of imperfect simultaneous channel identification, and then the imperfect scheduled user identification, with the latter is applicable when multiuser scheduling is exploited for desired link improvement. These two cases are addressed in the following two subsections.

\section{A. Imperfect Simultaneous Channel Identification}

The results in (14) and (15) are only applicable under perfect identification of the best possible channel per active user. These results need to be modified in order to quantify the variation in the statistical distribution of the received desired power by an active user when imprecise identification of the best possible interference-free channel occurs, as treated below.

From the order statistics that is given by $s_{\mathrm{D},(1), j} \leq$ $s_{\mathrm{D},(2), j} \leq \cdots \leq s_{\mathrm{D},\left(\left|\mathcal{B}_{j}\right|\right), j}$ and obtained by arranging $\left\{s_{\mathrm{D}, r, j}\right\}_{r=1}^{\left|\mathcal{B}_{j}\right|}$ in increasing order of magnitude, the $r^{*}$ th channel in order statistics may be identified to serve the $j$ th user, for $1 \leq r^{*} \leq\left|\mathcal{B}_{j}\right|$. The best scenario is when $r^{*}=\left|\mathcal{B}_{j}\right|$ at which $s_{\mathrm{D},\left(r^{*}\right), j}=\max _{r}\left\{s_{\mathrm{D}, r, j}\right\}_{r=1}^{\left|\mathcal{B}_{j}\right|}$, whereas the worst scenario occurs when $r^{*}=1$, which corresponds to $s_{\mathrm{D},\left(r^{*}\right), j}=$ $\min _{r}\left\{s_{\mathrm{D}, r, j}\right\}_{r=1}^{\left|\mathcal{B}_{j}\right|}$.

When the $r^{*}$ th channel in order statistics is identified, the statistics of the desired power on that imperfectly identified channel, which is referred to as $s_{\mathrm{D},\left(r^{*}\right), j, \widetilde{s e}}$, when $\left\{s_{\mathrm{D}, r, j}\right\}_{r=1}^{\left|\mathcal{B}_{j}\right|}$ are non-identically distributed, and capitalizing on the results from [35, Ch. 5], can be expressed as

$$
\begin{aligned}
\operatorname{Pr}\left\{s_{\mathrm{D},\left(r^{*}\right), j, \widetilde{\mathrm{se}}}<x|| \mathcal{B}_{j} \mid\right\} & =\sum_{\ell=r^{*}}^{\left|\mathcal{B}_{j}\right|} \sum_{S_{\ell}} \prod_{p=1}^{\ell} \operatorname{Pr}\left\{s_{\mathrm{D}, r_{p}, j}<x\right\} \\
& \times \prod_{p=\ell+1}^{\left|\mathcal{B}_{j}\right|}\left(1-\operatorname{Pr}\left\{s_{\mathrm{D}, r_{p}, j}<x\right\}\right) .
\end{aligned}
$$

The sum $S_{\ell}$ extends over all permutations $\left(r_{1}, r_{2}, \ldots, r_{\left|\mathcal{B}_{j}\right|}\right)$ of $1, \ldots,\left|\mathcal{B}_{j}\right|$, for which $r_{1}<r_{2}<\cdots<r_{\ell}$ and $r_{\ell+1}<$ $\cdots<r_{\left|\mathcal{B}_{j}\right|}$. For the special case of identically distributed $\left\{s_{\mathrm{D}, r, j}\right\}_{r=1}^{\left|\mathcal{B}_{j}\right|}$, the result in (26) reduces to

$$
\begin{aligned}
\operatorname{Pr}\left\{s_{\mathrm{D},\left(r^{*}\right), j, \widetilde{\mathrm{e}}}<x|| \mathcal{B}_{j} \mid\right\} & =\sum_{\ell=r^{*}}^{\left|\mathcal{B}_{j}\right|}\left(\begin{array}{c}
\left|\mathcal{B}_{j}\right| \\
\ell
\end{array}\right)\left[\operatorname{Pr}\left\{s_{\mathrm{D}, r, j}<x\right\}\right]^{\ell} \\
& \times\left[1-\operatorname{Pr}\left\{s_{\mathrm{D}, r, j}<x\right\}\right]^{\left|\mathcal{B}_{j}\right|-\ell} .
\end{aligned}
$$

An interesting example from the preceding results is stated herein to quantify the impact of imperfect identification of the best channel. Specifically, considering the statistics of the desired power under perfect best channel identification (i.e., case of $\left.r^{*}=\left|\mathcal{B}_{j}\right|\right)$ relative to that with second best channel 


$$
\operatorname{Pr}\left\{\left|\widetilde{\mathcal{B}}_{j}\right|=b \mid J>L_{j}-1\right\}=\left\{\begin{array}{l}
\prod_{m=1}^{M} \prod_{k=1}^{K} \prod_{n=1}^{N}\left(1-\operatorname{Pr}\left\{s_{\mathrm{I}, m, k, n, j} \rightarrow 0 \& \text { unshared }\right\}\right), \quad b=0 \\
\prod_{m=1}^{M} \prod_{k=1}^{K} \prod_{n=1}^{N}\left(1-\operatorname{Pr}\left\{s_{\mathrm{I}, m, k, n, j} \rightarrow 0 \& \text { unshared }\right\}\right) \\
\times \sum_{\mathcal{P} \in \mathcal{G}_{b}} \prod_{(m, k, n) \in \mathcal{P}}\left(\frac{\operatorname{Pr}\left\{s_{\mathrm{I}, m, k, n, j} \rightarrow 0 \& \text { unshared }\right\}}{\left(1-\operatorname{Pr}\left\{s_{\mathrm{I}, m, k, n, j} \rightarrow 0 \text { \& unshared }\right\}\right)}\right), \quad b=1,2, \ldots, M N K .
\end{array}\right.
$$

identification (i.e., case of $r^{*}=\left|\mathcal{B}_{j}\right|-1$ ), the result in (27) gives

$$
\begin{aligned}
& \operatorname{Pr}\left\{s_{\mathrm{D},\left(\left|\mathcal{B}_{j}\right|\right), j, \widetilde{\mathrm{se}}}<x|| \mathcal{B}_{j} \mid\right\}=\left[\operatorname{Pr}\left\{s_{\mathrm{D}, r, j}<x\right\}\right]^{\left|\mathcal{B}_{j}\right|}, \\
& \operatorname{Pr}\left\{s_{\mathrm{D},\left(\left|\mathcal{B}_{j}\right|-1\right), j, \widetilde{\mathrm{se}}}<x|| \mathcal{B}_{j} \mid\right\} \\
& =\left[\operatorname{Pr}\left\{s_{\mathrm{D}, r, j}<x\right\}\right]^{\left|\mathcal{B}_{j}\right|}+\left|\mathcal{B}_{j}\right|\left[\operatorname{Pr}\left\{s_{\mathrm{D}, r, j}<x\right\}\right]^{\left|\mathcal{B}_{j}\right|-1} \\
& \times\left[1-\operatorname{Pr}\left\{s_{\mathrm{D}, r, j}<x\right\}\right] .
\end{aligned}
$$

Therefore, for a given $\left|\mathcal{B}_{j}\right|$, it can be written that

$$
\begin{aligned}
& \frac{\operatorname{Pr}\left\{s_{\mathrm{D},\left(\left|\mathcal{B}_{j}\right|-1\right), j, \widetilde{\mathrm{se}}}<x|| \mathcal{B}_{j} \mid\right\}}{\operatorname{Pr}\left\{s_{\mathrm{D},\left(\left|\mathcal{B}_{j}\right|\right), j, \widetilde{\mathrm{se}}}<x|| \mathcal{B}_{j} \mid\right\}} \\
& =\left(1+\left|\mathcal{B}_{j}\right| \frac{\left.1-\operatorname{Pr}\left\{s_{\mathrm{D}, r, j}<x\right\}\right]}{\operatorname{Pr}\left\{s_{\mathrm{D}, r, j}<x\right\}}\right) \\
& \cong \begin{cases}1, & \operatorname{Pr}\left\{s_{\mathrm{D}, r, j}<x\right\} \rightarrow 1 ; \\
\left|\mathcal{B}_{j}\right|\left(\operatorname{Pr}\left\{s_{\mathrm{D}, r, j}<x\right\}\right)^{-1}, & \operatorname{Pr}\left\{s_{\mathrm{D}, r, j}<x\right\} \ll 1,\end{cases}
\end{aligned}
$$

which clarifies the associated loss when the second best channel is identified instead of the best channel. This loss is proportional to $\left|\mathcal{B}_{j}\right|$, irrespective to the source of imperfectness. It is also inversely proportional to the threshold $x$ in (29), wherein the loss is maximum when $x \rightarrow 0^{+}$, and it diminishes when $x \rightarrow+\infty$, at which the order statistics has no advantage.

The unconditional statistics of $s_{\mathrm{D}, j, \widetilde{\mathrm{se}}}$ for all possible cases of $\left|\mathcal{B}_{j}\right|$ can be obtained by combining the results in (26) or (27) with (10) or (11). In particular, for the case of $J \leq L_{j}-1$, using (10) into (26) and (27), the statistics of $s_{\mathrm{D}, j, \widetilde{\mathrm{se}}}$ follow the same forms as given in (26) and (27) with $\left|\mathcal{B}_{j}\right|$ therein is replaced by $M N K$ (i.e., case when all available channels are found to be interference-free). Although this particular case results in the maximum possible gain under perfect identification of the $j$ th user's desired channel, mainly due to having the maximum possible cardinality of $\mathcal{B}_{j}$, it will create the largest loss in the case of imperfect best channel identification, which can be clearly read from (29) for the case of second best channel identification scenario, wherein $\left|\mathcal{B}_{j}\right|=M N K$.

On the other hand, for the case of $J>L_{j}-1$, using the results in (11) into that in (26) gives

$$
\begin{aligned}
& \operatorname{Pr}\left\{s_{\mathrm{D},\left(\mathbf{r}^{*}\right), j, \widetilde{\mathrm{se}}}<x \mid J>L_{j}-1\right\} \\
& =\frac{1}{1-\operatorname{Pr}\left\{\left|\mathcal{B}_{j}\right|=0\right\}} \sum_{b=1}^{M N K}\left(\sum_{\ell_{b}=r_{b}^{*}}^{b} \sum_{S_{\ell_{b}}} \prod_{p=1}^{\ell_{b}} \operatorname{Pr}\left\{s_{\mathrm{D}, r_{p}, j}<x\right\}\right. \\
& \left.\times \prod_{p=\ell_{b}+1}^{b}\left(1-\operatorname{Pr}\left\{s_{\mathrm{D}, r_{p}, j}<x\right\}\right)\right) \operatorname{Pr}\left\{\left|\mathcal{B}_{j}\right|=b\right\},
\end{aligned}
$$

where $\left(\mathbf{r}^{*}\right)=\left[\left(r_{1}^{*}\right)\left(r_{2}^{*}\right) \cdots\left(r_{M N K}^{*}\right)\right]$ is a vector of length $M N K$ that includes the indexes of channels identified based on order statistics for any $\left|\mathcal{B}_{j}\right|$, with $1 \leq r_{b}^{*} \leq b$, for $b=$ $1,2, \ldots, M N K$. The result in (30) captures all possible events of the cardinality of interference-free channels, $\left|\mathcal{B}_{j}\right|$, and the imperfectness in channel identification for each event. Specifically, the index of the identified channel in order statistics is $r_{b}^{*}$, and hence, the imperfectness in channel identification of the $j$ th user can be different for different occasions of $\left|\mathcal{B}_{j}\right|=b$. Under perfect best channel identification for all possible values of $\left|\mathcal{B}_{j}\right|$, the result in (30) reduces to that in (15). Moreover, when $\left\{s_{\mathrm{D}, r, j}\right\}_{r=1}^{\left|\mathcal{B}_{j}\right|}$ for any $\left|\mathcal{B}_{j}\right|$ are identically distributed, the result in (30) reduces to

$$
\begin{aligned}
& \operatorname{Pr}\left\{s_{\mathrm{D},\left(\mathbf{r}^{*}\right), j, \widetilde{s e}}<x \mid J>L_{j}-1\right\} \\
& =\frac{1}{1-\operatorname{Pr}\left\{\left|\mathcal{B}_{j}\right|=0\right\}} \sum_{b=1}^{M N K}\left(\sum_{\ell_{b}=r_{b}^{*}}^{b}\left(\begin{array}{c}
b \\
\ell_{b}
\end{array}\right)\left[\operatorname{Pr}\left\{s_{\mathrm{D}, r, j}<x\right\}\right]^{\ell_{b}}\right. \\
& \left.\times\left[1-\operatorname{Pr}\left\{s_{\mathrm{D}, r, j}<x\right\}\right]^{b-\ell_{b}}\right) \operatorname{Pr}\left\{\left|\mathcal{B}_{j}\right|=b\right\}
\end{aligned}
$$

To provide a quantitative view about the effect of imperfect channel identification for all possible events of $\left|\mathcal{B}_{j}\right|$, consider the result in (31) with best channel identification and second best channel identification, both for any value of $\left|\mathcal{B}_{j}\right|$. For the first case, it follows that $r_{b}^{*}=b$, for $b=1,2, \ldots, M N K$, and therefore the vector $\left(\mathbf{r}^{*}\right) \triangleq(\mathbf{b})=\left[\begin{array}{llll}(1) & (2) & (3) & \cdots\end{array}(M N K)\right]$, whereas for the second case, it follows that $r_{b}^{*}=b-1$, for $b=2, \ldots, M N K$, and hence, $\left(\mathbf{r}^{*}\right) \triangleq(\mathbf{b}-1)=$ [(1) (1) (2) $\cdots(M N K-1)]$ (note that the event $\left|\mathcal{B}_{j}\right|=1$ does not involve order statistics). Using the preceding results in (31) give

$$
\begin{aligned}
& \operatorname{Pr}\left\{s_{\mathrm{D},(\mathbf{b}), j, \widetilde{\mathrm{se}}}<x \mid J>L_{j}-1\right\} \\
& =\frac{1}{1-\operatorname{Pr}\left\{\left|\mathcal{B}_{j}\right|=0\right\}} \sum_{b=1}^{M N K}\left(\left[\operatorname{Pr}\left\{s_{\mathrm{D}, r, j}<x\right\}\right]^{b}\right) \operatorname{Pr}\left\{\left|\mathcal{B}_{j}\right|=b\right\} \\
& \operatorname{Pr}\left\{s_{\mathrm{D},(\mathbf{b}-1), j, \widetilde{\mathrm{se}}}<x \mid J>L_{j}-1\right\} \\
& =\frac{1}{1-\operatorname{Pr}\left\{\left|\mathcal{B}_{j}\right|=0\right\}} \sum_{b=1}^{M N K}\left(\left[\operatorname{Pr}\left\{s_{\mathrm{D}, r, j}<x\right\}\right]^{b}\right. \\
& \left.+b\left[\operatorname{Pr}\left\{s_{\mathrm{D}, r, j}<x\right\}\right]^{b-1}\left[1-\operatorname{Pr}\left\{s_{\mathrm{D}, r, j}<x\right\}\right]\right) \operatorname{Pr}\left\{\left|\mathcal{B}_{j}\right|=b\right\}
\end{aligned}
$$


Therefore, it can be shown, based on (32), that

$$
\begin{aligned}
& \frac{\operatorname{Pr}\left\{s_{\mathrm{D},(\mathbf{b}-1), j, \widetilde{\mathrm{se}}}<x \mid J>L_{j}-1\right\}}{\operatorname{Pr}\left\{s_{\mathrm{D},(\mathbf{b}), j, \widetilde{\mathrm{se}}}<x \mid J>L_{j}-1\right\}} \\
& =1+\frac{1}{1-\operatorname{Pr}\left\{\left|\mathcal{B}_{j}\right|=0\right\}} \\
& \times \sum_{b=1}^{M N K}\left(b \frac{1-\operatorname{Pr}\left\{s_{\mathrm{D}, r, j}<x\right\}}{\operatorname{Pr}\left\{s_{\mathrm{D}, r, j}<x\right\}}\right) \operatorname{Pr}\left\{\left|\mathcal{B}_{j}\right|=b\right\} \\
& =1+\mathbb{E}\left\{\left|\mathcal{B}_{j}\right|\right\} \frac{1-\operatorname{Pr}\left\{s_{\mathrm{D}, r, j}<x\right\}}{\operatorname{Pr}\left\{s_{\mathrm{D}, r, j}<x\right\}} \\
& \cong\left\{\begin{array}{l}
1, \\
\mathbb{E}\left\{\left|\mathcal{B}_{j}\right|\right\}\left(\operatorname{Pr}\left\{s_{\mathrm{D}, r, j}<x\right\}\right)^{-1}, \quad \operatorname{Pr}\left\{s_{\mathrm{D}, r, j}<x\right\} \ll 1 .
\end{array}\right.
\end{aligned}
$$

The results in (33) reveal that the loss due to second best channel identification is proportional to the expected value of $\left|\mathcal{B}_{j}\right|$, denoted by $\mathbb{E}\left\{\left|\mathcal{B}_{j}\right|\right\} \triangleq \frac{1}{1-\operatorname{Pr}\left\{\left|\mathcal{B}_{j}\right|=0\right\}} \sum_{b=1}^{M N K} b \operatorname{Pr}\left\{\left|\mathcal{B}_{j}\right|=\right.$ b\}. A higher value of $\mathbb{E}\left\{\left|\mathcal{B}_{j}\right|\right\}$ is a desirable event in channel identification under perfect condition, but it incurs more losses under imperfect identification. However, the mount of loss in this case is generally lower than that noticed when $J \leq L_{j}-1$ since $\mathbb{E}\left\{\left|\mathcal{B}_{j}\right|\right\} \leq M N K$.

\section{B. Imperfect User Identification}

Subsection IV-B treats perfect identification of the scheduled user under perfect best channel identification for each active user involved in multiuser scheduling. However, the identification of the scheduled user may be imprecise, independently of whether the channel identification per active user is perfect or imperfect as discussed in subsections IV-A and V-A, respectively.

1) Specific Number of Scheduled Users: From the findings in the preceding subsection, let $s_{\mathrm{D},\left(r^{*}\right),(1), \widetilde{\mathrm{se}}} \leq s_{\mathrm{D},\left(r^{*}\right),(2), \widetilde{\mathrm{se}}} \leq$ $\cdots \leq s_{\mathrm{D},\left(r^{*}\right),\left(\left|\mathcal{D}_{\ell}\right|\right), \widetilde{\mathrm{se}}}$ be the order statistics that is obtained by arranging $\left\{s_{\mathrm{D},\left(r^{*}\right), j, \widetilde{\text { se }}}\right\}_{j=1}^{\left|\mathcal{D}_{\ell}\right|}$ in increasing order of magnitude. Note that the marginal statistics of $\left\{s_{\mathrm{D},\left(r^{*}\right), j, \widetilde{\text {, e }}}\right\}_{j=1}^{\left|\mathcal{D}_{\ell}\right|}$ are given in (26) and (27) with $\left|\mathcal{B}_{j}\right|=M N K$ for the case of $J \leq L_{j}-1$. Moreover, let $s_{\mathrm{D},\left(\mathbf{r}^{*}\right),(1), \widetilde{\mathrm{se}}} \leq s_{\mathrm{D},\left(\mathbf{r}^{*}\right),(2), \widetilde{\mathrm{se}}} \leq$ $\cdots \leq s_{\mathrm{D},\left(\mathbf{r}^{*}\right),\left(\left|\mathcal{D}_{\ell}\right|\right), \widetilde{\mathrm{se}}}$ be the order statistics that is obtained by arranging $\left\{s_{\mathrm{D},\left(\mathbf{r}^{*}\right), j, \widetilde{\mathrm{e}}}\right\}_{j=1}^{\left|\mathcal{D}_{\ell}\right|}$ in increasing order of magnitude, where the marginal statistics of $\left\{s_{\mathrm{D},\left(\mathbf{r}^{*}\right), j, \widetilde{\mathrm{se}}}\right\}_{j=1}^{\left|\mathcal{D}_{\ell}\right|}$ are given in (30) and (31) for the case of $J>L_{j}-1$. Then the $j^{*}$ th user in the order statistics, for $1 \leq j^{*} \leq\left|\mathcal{D}_{\ell}\right|$, may be allocated that $\ell$ th channel.

According to the order statistics presented above, the best possible user scheduling from desired link improvement perspective takes place when $j^{*}=\left|\mathcal{D}_{\ell}\right|$, at which $s_{\mathrm{D},\left(r^{*}\right),\left(j^{*}\right), \widetilde{\mathrm{se}}}=$ $\max _{j}\left\{s_{\mathrm{D},\left(r^{*}\right), j, \widetilde{\mathrm{se}}}\right\}_{j=1}^{\left|\mathcal{D}_{\ell}\right|}$ for $J \leq L_{j}-1$ or $s_{\mathrm{D},\left(\mathbf{r}^{*}\right),\left(j^{*}\right), \widetilde{\mathrm{se}}}=$ $\max _{j}\left\{s_{\mathrm{D},\left(\mathbf{r}^{*}\right), j, \widetilde{\mathrm{se}}}\right\}_{j=1}^{\left|\mathcal{D}_{\ell}\right|}$ for $J>L_{j}-1$. On the other hand, the worst user scheduling scenario happens when $j^{*}=1$, at which $s_{\mathrm{D},\left(r^{*}\right),\left(j^{*}\right), \widetilde{\mathrm{se}}}=\min _{j}\left\{s_{\mathrm{D},\left(r^{*}\right), j, \widetilde{\mathrm{se}}}\right\}_{j=1}^{\left|\mathcal{D}_{\ell}\right|}$ for $J \leq L_{j}-1$ or $s_{\mathrm{D},\left(\mathbf{r}^{*}\right),\left(j^{*}\right), \widetilde{\mathrm{se}}}=\min _{j}\left\{s_{\mathrm{D},\left(\mathbf{r}^{*}\right), j, \widetilde{\mathrm{se}}}\right\}_{j=1}^{\left|\mathcal{D}_{\ell}\right|}$ for $J>L_{j}-1$.

When the $j^{*}$ th user is identified by the scheduler, the statistics of the desired power of the scheduled user, which is referred to as $s_{\mathrm{D},\left(r^{*}\right),\left(j^{*}\right), \widetilde{\mathrm{se}}}$ for $J \leq L_{j}-1$, can be expressed as

$$
\begin{aligned}
\operatorname{Pr}\left\{s_{\mathrm{D},\left(r^{*}\right),\left(j^{*}\right), \widetilde{\mathrm{se}}}<x|| \mathcal{D}_{\ell} \mid\right\} & =\sum_{c=j^{*}}^{\left|\mathcal{D}_{\ell}\right|} \sum_{S_{c}} \prod_{p=1}^{c} \operatorname{Pr}\left\{s_{\mathrm{D},\left(r^{*}\right), j_{p}, \widetilde{\mathrm{se}}}<x\right\} \\
& \times \prod_{p=c+1}^{\left|\mathcal{D}_{\ell}\right|}\left(1-\operatorname{Pr}\left\{s_{\mathrm{D},\left(r^{*}\right), j_{p}, \widetilde{\mathrm{se}}}<x\right\}\right),
\end{aligned}
$$

where $\operatorname{Pr}\left\{s_{\mathrm{D},\left(r^{*}\right), j_{p}, \widetilde{\mathrm{se}}}<x\right\}$ is defined in (26) and for a special case in (27) with $\left|\mathcal{B}_{j}\right|=M N K$. The sum $S_{c}$ extends over all permutations $\left(j_{1}, j_{2}, \ldots, j_{\left|\mathcal{D}_{\ell}\right|}\right)$ of $1, \ldots,\left|\mathcal{D}_{\ell}\right|$, for which $j_{1}<$ $j_{2}<\cdots<j_{c}$ and $j_{c+1}<\cdots<j_{\left|\mathcal{D}_{\ell}\right|}$. For the special case when $\left\{\operatorname{Pr}\left\{s_{\mathrm{D},\left(r^{*}\right), j, \widetilde{\text { se }}}<x\right\}\right\}_{j=1}^{\left|\mathcal{D}_{\ell}\right|}$ are identically distributed, the result in (34) reduces to

$$
\begin{aligned}
\operatorname{Pr}\left\{s_{\mathrm{D},\left(r^{*}\right),\left(j^{*}\right), \widetilde{\mathrm{se}}}<x|| \mathcal{D}_{\ell} \mid\right\} & =\sum_{c=j^{*}}^{\left|\mathcal{D}_{\ell}\right|}\left(\begin{array}{c}
\left|\mathcal{D}_{\ell}\right| \\
c
\end{array}\right)\left[\operatorname{Pr}\left\{s_{\mathrm{D},\left(r^{*}\right), j, \widetilde{\mathrm{se}}}<x\right\}\right]^{c} \\
& \times\left[1-\operatorname{Pr}\left\{s_{\mathrm{D},\left(r^{*}\right), j, \widetilde{\mathrm{se}}}<x\right\}\right]^{\left|\mathcal{D}_{\ell}\right|-c}
\end{aligned}
$$

where $\operatorname{Pr}\left\{s_{\mathrm{D},\left(r^{*}\right), j, \widetilde{\mathrm{se}}}<x\right\}$ is also given by (26) and for a special case in (27) with $\left|\mathcal{B}_{j}\right|=M N K$.

Now, for $J>L_{j}-1$, the statistics of the desired power of the scheduled user, which is denoted by $s_{\mathrm{D},\left(\mathbf{r}^{*}\right),\left(j^{*}\right), \widetilde{\text { se }}}$, can be written as

$$
\begin{aligned}
\operatorname{Pr}\left\{s_{\mathrm{D},\left(\mathbf{r}^{*}\right),\left(j^{*}\right), \widetilde{\mathrm{se}}}<x|| \mathcal{D}_{\ell} \mid\right\} & =\sum_{c=j^{*}}^{\left|\mathcal{D}_{\ell}\right|} \sum_{S_{c}} \prod_{p=1}^{c} \operatorname{Pr}\left\{s_{\mathrm{D},\left(\mathbf{r}^{*}\right), j_{p}, \widetilde{\mathrm{se}}}<x\right\} \\
\times & \prod_{p=c+1}^{\left|\mathcal{D}_{\ell}\right|}\left(1-\operatorname{Pr}\left\{s_{\mathrm{D},\left(\mathbf{r}^{*}\right), j_{p}, \widetilde{\mathrm{se}}}<x\right\}\right) .
\end{aligned}
$$

For the special case when $\left\{\operatorname{Pr}\left\{s_{\mathrm{D},\left(\mathbf{r}^{*}\right), j, \widetilde{\mathrm{se}}}<x\right\}\right\}_{j=1}^{\left|\mathcal{D}_{\ell}\right|}$ are identically distributed, (36) reduces to

$$
\begin{aligned}
\operatorname{Pr}\left\{s_{\mathrm{D},\left(\mathbf{r}^{*}\right),\left(j^{*}\right), \widetilde{\mathrm{se}}}<x|| \mathcal{D}_{\ell} \mid\right\} & =\sum_{c=j^{*}}^{\left|\mathcal{D}_{\ell}\right|}\left(\begin{array}{c}
|\mathcal{D}| \\
c
\end{array}\right)\left[\operatorname{Pr}\left\{s_{\mathrm{D},\left(\mathbf{r}^{*}\right), j, \widetilde{\mathrm{se}}}<x\right\}\right]^{c} \\
& \times\left[1-\operatorname{Pr}\left\{s_{\mathrm{D},\left(\mathbf{r}^{*}\right), j, \widetilde{\mathrm{se}}}<x\right\}\right]^{\left|\mathcal{D}_{\ell}\right|-c},
\end{aligned}
$$

where $\operatorname{Pr}\left\{s_{\mathrm{D},\left(\mathbf{r}^{*}\right), j, \widetilde{\text { se }}}<x\right\}$ is given in (30) and for a special case in (31).

Note that (34)-(37) address the scenario when the imperfect channel identification is similar for all scheduled users. However, the variation in the prediction accuracy of the suitable channel among users can be also addressed. Particularly, when 
the scheduler identifies the best active user perfectly, it follows that

$$
\begin{aligned}
& \operatorname{Pr}\left\{s_{\mathrm{D},\left(r_{1}^{*}\right), \cdots,\left(r_{\left|\mathcal{D}_{\ell}\right|}^{*}\right),\left(\left|\mathcal{D}_{\ell}\right|\right), \widetilde{\mathrm{se}}}<x|| \mathcal{D}_{\ell} \mid\right\} \\
& =\prod_{j=1}^{\left|\mathcal{D}_{\ell}\right|} \operatorname{Pr}\left\{s_{\mathrm{D},\left(r_{j}^{*}\right), j, \widetilde{\mathrm{se}}}<x\right\}, J \leq L_{j}-1 ; \\
& \operatorname{Pr}\left\{s_{\mathrm{D},\left(\mathbf{r}_{1}^{*}\right), \cdots,\left(\mathbf{r}_{\left|\mathcal{D}_{\ell}\right|}^{*}\right),\left(\left|\mathcal{D}_{\ell}\right|\right), \widetilde{\mathrm{se}}}<x|| \mathcal{D}_{\ell} \mid\right\} \\
& =\prod_{j=1}^{\left|\mathcal{D}_{\ell}\right|} \operatorname{Pr}\left\{s_{\mathrm{D},\left(\mathbf{r}_{j}^{*}\right), j, \widetilde{\mathrm{se}}}<x\right\}, J>L_{j}-1,
\end{aligned}
$$

where $\left(r_{1}^{*}\right), \cdots,\left(r_{\left|\mathcal{D}_{\ell}\right|}^{*}\right)$ and $\left(\mathbf{r}_{1}^{*}\right), \cdots,\left(\mathbf{r}_{\left|\mathcal{D}_{\ell}\right|}^{*}\right)$ denote the order statistics ranking during the channel identification stage for active users whose indexes belong to the set $\mathcal{D}_{\ell}$ for the cases of $J \leq L_{j}-1$ and $J>L_{j}-1$, respectively.

2) Limiting Cases: In this part, some limiting cases of the scenarios described in the previous part, which carry practical importance, are treated. Specifically, using (27) for the case of $J \leq L_{j}-1$ with $\left|\mathcal{B}_{j}\right|=M N K$ in (35) results in (39) on the top of the next page. An important limiting case from (39) takes place under perfect channel identification per active user, for which (39) gives

$$
\begin{aligned}
& \operatorname{Pr}\left\{s_{\mathrm{D},(M N K),\left(j^{*}\right), \widetilde{\mathrm{se}}}<x|| \mathcal{D}_{\ell} \mid\right\} \\
& =\sum_{c=j^{*}}^{\left|\mathcal{D}_{\ell}\right|}\left(\begin{array}{c}
\left|\mathcal{D}_{\ell}\right| \\
c
\end{array}\right)\left[\left[\operatorname{Pr}\left\{s_{\mathrm{D}, r, j}<x\right\}\right]^{M N K}\right]^{c} \\
& \times\left[1-\left[\operatorname{Pr}\left\{s_{\mathrm{D}, r, j}<x\right\}\right]^{M N K}\right]^{\left|\mathcal{D}_{\ell}\right|-c} .
\end{aligned}
$$

Based on (40), considering the statistics of the desired power with best user identification, which gives $\operatorname{Pr}\left\{s_{\mathrm{D},(M N K),\left(\left|\mathcal{D}_{\ell}\right|\right), \widetilde{\mathrm{se}}}<x|| \mathcal{D}_{\ell} \mid\right\}$, and that with second best user identification, which results in $\operatorname{Pr}\left\{s_{\mathrm{D},(M N K),\left(\left|\mathcal{D}_{\ell}\right|-1\right), \widetilde{\mathrm{se}}}<\right.$ $\left.x|| \mathcal{D}_{\ell} \mid\right\}$, it can be shown that

$$
\begin{aligned}
& \frac{\operatorname{Pr}\left\{s_{\mathrm{D},(M N K),\left(\left|\mathcal{D}_{\ell}\right|-1\right), \widetilde{\mathrm{se}}}<x|| \mathcal{D}_{\ell} \mid\right\}}{\operatorname{Pr}\left\{s_{\mathrm{D},(M N K),\left(\left|\mathcal{D}_{\ell}\right|\right), \widetilde{\mathrm{se}}}<x|| \mathcal{D}_{\ell} \mid\right\}} \\
& =\left(1+\left|\mathcal{D}_{\ell}\right| \frac{1-\left[\operatorname{Pr}\left\{s_{\mathrm{D}, r, j}<x\right\}\right]^{M N K}}{\left[\operatorname{Pr}\left\{s_{\mathrm{D}, r, j}<x\right\}\right]^{M N K}}\right) \\
& \cong \begin{cases}1, & \operatorname{Pr}\left\{s_{\mathrm{D}, r, j}<x\right\} \rightarrow 1 ; \\
\left|\mathcal{D}_{\ell}\right|\left(\operatorname{Pr}\left\{s_{\mathrm{D}, r, j}<x\right\}\right)^{-M N K}, & \operatorname{Pr}\left\{s_{\mathrm{D}, r, j}<x\right\} \ll 1,\end{cases}
\end{aligned}
$$

which clarifies that the imperfectness in the scheduled user index results in a linearly increasing loss as a function of the cardinality of scheduled users $\left|\mathcal{D}_{\ell}\right|$ and an exponentially increasing loss as a function of the cardinality of the set of interference-free channels per active user, which is herein $\left|\mathcal{B}_{j}\right|=M N K$. This exponential increase in the associated loss can be explained by noting that the user scheduling process occurs after perfect identification of the best channel per active user, which have been obtained via searching over the maximum possible cardinality of interference-free channels.

Another important limiting case from (39) is deduced when the identification of the best possible interference-free channel per active user is imperfect while the multiuser scheduler precisely identifies the best active user. In this case, the resulting statistics of the desired power of the scheduled user can be written as

$$
\begin{aligned}
& \operatorname{Pr}\left\{s_{\mathrm{D},\left(r^{*}\right),\left(\left|\mathcal{D}_{\ell}\right|\right), \widetilde{\mathrm{se}}}<x|| \mathcal{D}_{\ell} \mid\right\} \\
& =\left[\sum_{h=r^{*}}^{M N K}\left(\begin{array}{c}
M N K \\
h
\end{array}\right)\left[\operatorname{Pr}\left\{s_{\mathrm{D}, r, j}<x\right\}\right]^{h}\right. \\
& \left.\times\left[1-\operatorname{Pr}\left\{s_{\mathrm{D}, r, j}<x\right\}\right]^{M N K-h}\right]^{\left|\mathcal{D}_{\ell}\right|} .
\end{aligned}
$$

Considering the statistics of the desired power of the best scheduled user for the two cases of best interferencefree channel identification, $\operatorname{Pr}\left\{s_{\mathrm{D},(M N K),\left(\left|\mathcal{D}_{\ell}\right|\right), \widetilde{\mathrm{se}}}<x|| \mathcal{D}_{\ell} \mid\right\}$, and second best interference-free channel identification, $\operatorname{Pr}\left\{s_{\mathrm{D},(M N K-1),\left(\left|\mathcal{D}_{\ell}\right|\right), \widetilde{\mathrm{se}}}<x|| \mathcal{D}_{\ell} \mid\right\}$, it can be shown that

$$
\begin{aligned}
& \frac{\operatorname{Pr}\left\{s_{\mathrm{D},(M N K-1),(|\mathcal{D}|), \widetilde{\mathrm{se}}}<x|| \mathcal{D}_{\ell} \mid\right\}}{\operatorname{Pr}\left\{s_{\mathrm{D},(M N K),(|\mathcal{D}|), \widetilde{\mathrm{se}}}<x|| \mathcal{D}_{\ell} \mid\right\}} \\
& =\left(1+M N K \frac{1-\operatorname{Pr}\left\{s_{\mathrm{D}, r, j}<x\right\}}{\operatorname{Pr}\left\{s_{\mathrm{D}, r, j}<x\right\}}\right)^{\left|\mathcal{D}_{\ell}\right|} \\
& \cong \begin{cases}1, & \operatorname{Pr}\left\{s_{\mathrm{D}, r, j}<x\right\} \rightarrow 1 ; \\
\left(\frac{M N K}{\operatorname{Pr}\left\{s_{\mathrm{D}, r, j}<x\right\}}\right)^{\left|\mathcal{D}_{\ell}\right|}, & \operatorname{Pr}\left\{s_{\mathrm{D}, r, j}<x\right\} \ll 1,\end{cases}
\end{aligned}
$$

which shows that the loss encountered due to the effect of imperfect channel identification per active user that is involved in multiuser scheduling increases linearly with the cardinality of interference-free channels, $\left|\mathcal{B}_{j}\right|=M N K$, and exponentially with the cardinality of scheduled users, $\left|\mathcal{D}_{\ell}\right|$. Note that the loss between two brackets following the first equity sign in (43) is exactly the loss observed by each user due to imperfectness in channel identification (refer to (29)). The total loss under perfect best user scheduling is therefore the multiplication of the individual losses per individual users. An important application of this observation is the case when only $\beta \leq\left|\mathcal{D}_{\ell}\right|$ number of active users, each of which achieves best interference-free channel identification, while the remaining $\left|\mathcal{D}_{\ell}\right|-\beta$ number of active users encounter imperfect channel identification. In this case, the term $\left|\mathcal{D}_{\ell}\right|-\beta$ will replace the exponent $\left|\mathcal{D}_{\ell}\right|$ in (43), thereby indicating a reduced level of loss.

3) Results for Arbitrary Number of Scheduled Users: After considering all possible events of $\left|\mathcal{D}_{\ell}\right|$, the unconditional results of that in (34) and (36) for the cases of $J \leq L_{j}-1$ 


$$
\begin{aligned}
\operatorname{Pr}\left\{s_{\mathrm{D},\left(r^{*}\right),\left(j^{*}\right), \widetilde{\mathrm{se}}}<x|| \mathcal{D}_{\ell} \mid\right\} & =\sum_{c=j^{*}}^{\left|\mathcal{D}_{\ell}\right|}\left(\begin{array}{c}
\left|\mathcal{D}_{\ell}\right| \\
c
\end{array}\right)\left[\sum_{h=r^{*}}^{M N K}\left(\begin{array}{c}
M N K \\
h
\end{array}\right)\left[\operatorname{Pr}\left\{s_{\mathrm{D}, r, j}<x\right\}\right]^{h}\left[1-\operatorname{Pr}\left\{s_{\mathrm{D}, r, j}<x\right\}\right]^{M N K-h}\right]^{c} \\
& \times\left[1-\sum_{h=r^{*}}^{M N K}\left(\begin{array}{c}
M N K \\
h
\end{array}\right)\left[\operatorname{Pr}\left\{s_{\mathrm{D}, r, j}<x\right\}\right]^{h}\left[1-\operatorname{Pr}\left\{s_{\mathrm{D}, r, j}<x\right\}\right]^{M N K-h}\right]^{\left|\mathcal{D}_{\ell}\right|-c}
\end{aligned}
$$

and $J>L_{j}-1$ can be written as

$$
\begin{aligned}
\operatorname{Pr}\left\{s_{\mathrm{D}, \widetilde{\mathrm{se}}}<x\right\} & =\frac{1}{1-\operatorname{Pr}\left\{\left|\mathcal{D}_{\ell}\right|=0\right\}} \\
& \times \sum_{v_{0}=1}^{J_{0}}\left(\operatorname{Pr}\left\{s_{\mathrm{D},\left(r^{*}\right),\left(j^{*}\right), \widetilde{\mathrm{se}}}<x|| \mathcal{D}_{\ell} \mid\right\}\right) \\
& \times \operatorname{Pr}\left\{\left|\mathcal{D}_{\ell}\right|=v_{0}\right\}, \\
\operatorname{Pr}\left\{s_{\mathrm{D}, \widetilde{\mathrm{se}}}<x\right\} & =\frac{1}{1-\operatorname{Pr}\left\{\left|\mathcal{D}_{\ell}\right|=0\right\}} \\
& \times \sum_{v_{0}=1}^{J_{0}}\left(\operatorname{Pr}\left\{s_{\mathrm{D},\left(\mathbf{r}^{*}\right),\left(j^{*}\right), \widetilde{\mathrm{se}}}<x|| \mathcal{D}_{\ell} \mid\right\}\right) \\
& \times \operatorname{Pr}\left\{\left|\mathcal{D}_{\ell}\right|=v_{0}\right\} .
\end{aligned}
$$

The same methodology above can be also applied to other results in subsection V-B1, such as (35), (37), and (38). Moreover, they can be extended to study limiting cases similar to those shown in subsection V-B2. Further details are omitted herein for brevity.

\section{NUMERICAL EXAMPLES}

This section presents some numerical results to clarify the outcomes of the developed models in the previous sections. The presented numerical results are confirmed through simulations, which have been obtained by generating the underlying random quantities using specific distributions as described below $^{8}$ as well as according to the developed models. The results are averaged over 10 million channel realizations to achieve accurate computations.

For the sake of simplicity, all channels are assumed to be identically distributed with an average power gain of 0 $\mathrm{dBm}$. The fading distributions that are associated with different channels are modeled using Rayleigh distribution. The number of antennas at the receive station of each user is taken to be the same, which gives $L_{j}=L, \forall j^{9}$. Unless otherwise stated, the value of $p_{\text {act }}$ in (1) is taken to be 0.7 , and an active user is equally likely to access a specific channel. Moreover, $M N K$ refers to the total number of available channels, which can be distributed over number of physical channels $M$, number of transmit antenna per each AP $N$, and number of deployed AP $K$. For instance, when $M N K=5$, there can be 5 physical channels and single antenna at single deployed AP. The users are randomly placed according to a homogeneous poisson

\footnotetext{
${ }^{8}$ It is noted that the results in this paper are applicable for any user and AP placement distribution, and the simulation results shown herein are possible resulting examples.

${ }^{9}$ Note that no specific correlation model among receive antennas is needed since the receive antennas will not provide any diversity gain.
}

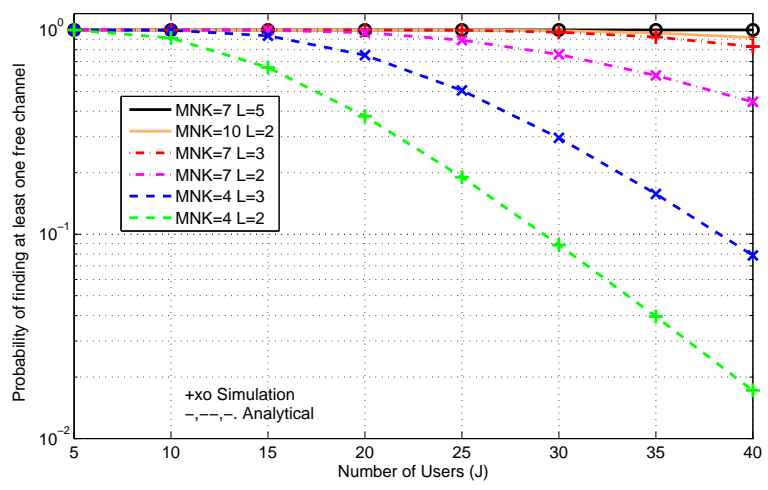

Fig. 2. Probability of finding at least one interference-free channel as a function of the number of users, $J$, with $M N K=4,7$, and 10, and $p_{\text {act }}=0.7$, and different sizes of the receive array per user, $L$.

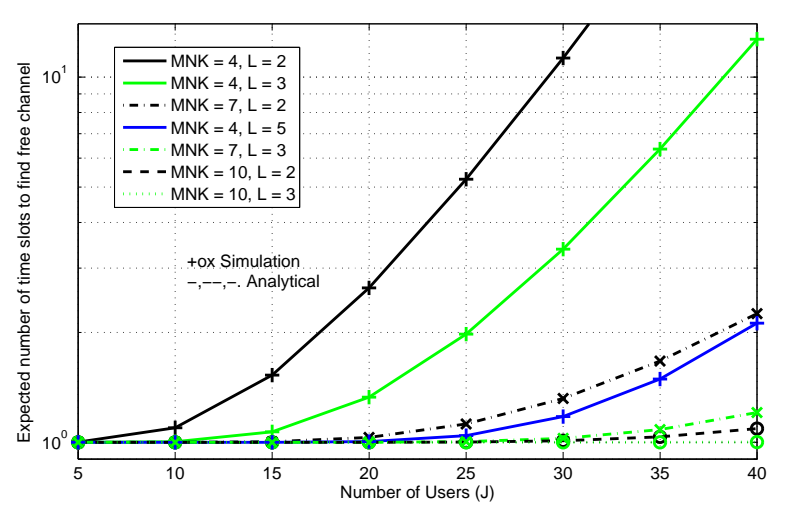

Fig. 3. Expected number of packet durations required to find at least one interference-free channel as a function of the number of users, $J$, with $M N K=4,7$, and 10 , and $p_{\text {act }}=0.7$.

point distribution, and the simulation runs are performed for a relatively large number of users locations to average out the effect of users' distribution. As the results are shown as a function of the number of users, the placement of the users is obtained from the uniform distribution. Each AP is assumed to be at the middle of its defined coverage space, which is related to the downlink average power.

Fig. 2 shows the probability of finding at least one interference-free channel, $\operatorname{Pr}\left\{\left|\mathcal{B}_{j}\right| \geq 1\right\}$, as a function of the number of users for different values of the number of available channels and size of receive array per each user with $p_{\text {act }}=0.7$. The results show that this probability decreases with the increase in the number of users, the decrease in the 


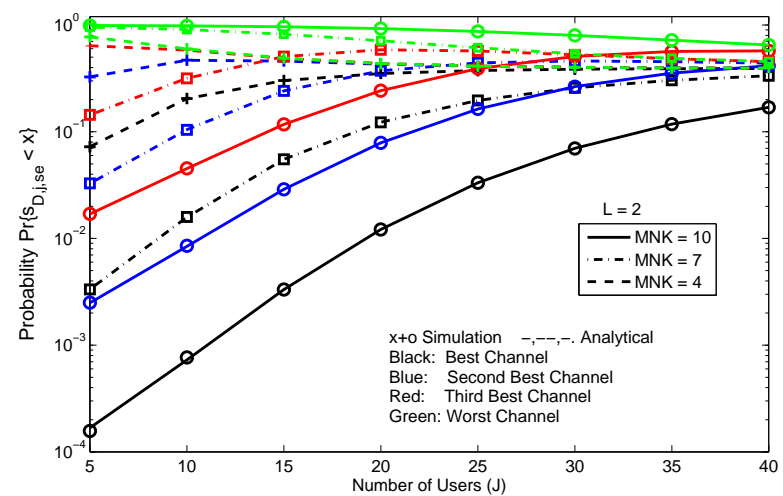

Fig. 4. Statistics of the received desired power on the best possible identified channel per active user, $\operatorname{Pr}\left\{s_{\mathrm{D}, j, \mathrm{se}}<x\right\}$ and $\operatorname{Pr}\left\{s_{\mathrm{D}, j, \widetilde{\mathrm{se}}}<x\right\}$ with $x=0.5$ as a function of the number of users, $J$, with $M N K=4,7$, and 10 , and $L=2$.

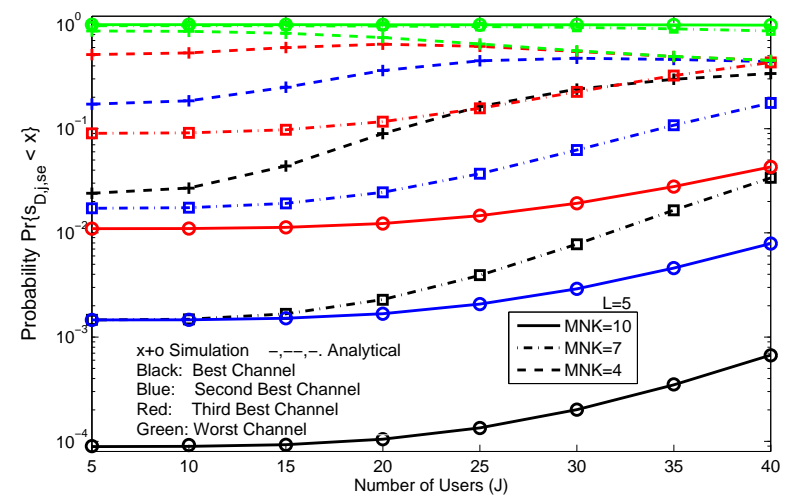

Fig. 5. Statistics of the received desired power associated on the best possible identified channel per active user, $\operatorname{Pr}\left\{s_{\mathrm{D}, j, \mathrm{se}}<x\right\}$ and $\operatorname{Pr}\left\{s_{\mathrm{D}, j, \widetilde{\mathrm{se}}}<x\right\}$ with $x=0.5$ as a function of the number of users, $J$, with $M N K=4,7$, and 10 , and $L=5$.

total number of available channels, and the decrease in the size of the receive array per each user. For instance, when $M N K=7$ and $L=5$, it is seen there will be at least one interference-free channel even when there are $J=40$ users.

Fig. 3 shows the expected number of packet durations needed by the $j$ th user to find at least one interference-free channel, $\mathbb{E}\left\{Y_{j}\right\}$. As the number of channels increases, it is more likely that at least one of them is interference-free. Similarly, with increase in the number of receive antennas, an active user can suppress higher number of interference sources. Moreover, with only a total of 10 channels and 3 receive antennas per active user, a user can find an interference-free channel in the first trial even when the number of users is $J=40$ and $p_{\text {act }}=0.7$. Also, the number of users and the expected number of packet durations to find an interferencefree channel are in a proportional relationship. However, as it has been observed that if there are enough available channels and active users have even a small number of receive antennas, the effect of the increase in $J$ can still be tolerated, and an interference-free channel identification is a likely event for any active user on average.

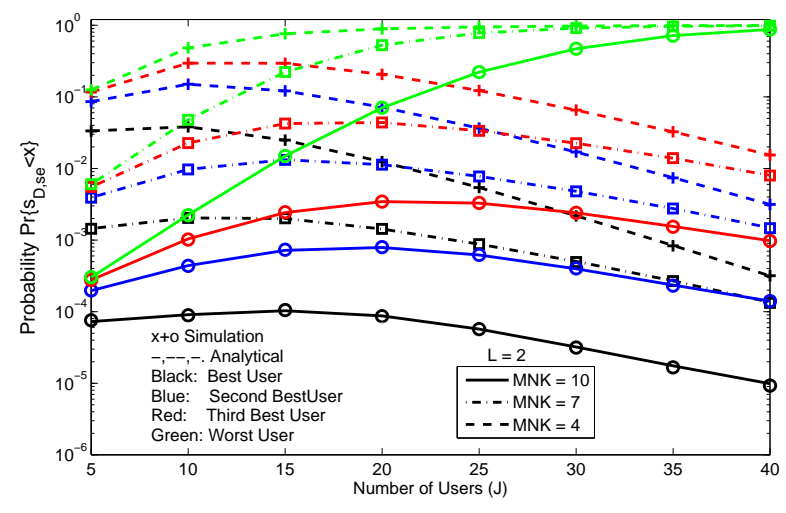

Fig. 6. Statistics of the scheduled user received desired power under perfect and imperfect scheduling, $\operatorname{Pr}\left\{s_{\mathrm{D}, \mathrm{se}}<x\right\}$ and $\operatorname{Pr}\left\{s_{\mathrm{D}, \widetilde{\mathrm{se}}}<x\right\}$ with $x=0.5$, and perfect channel identification per active user, as a function of the number of users $J=J_{0}$ with $M N K=4,7$, and 10 , and $L=2$.

Figs. 4 and 5 show $\operatorname{Pr}\left\{s_{\mathrm{D}, j, \text { se }}<x\right\}$ and $\operatorname{Pr}\left\{s_{\mathrm{D}, j, \widetilde{\mathrm{se}}}<x\right\}$ of the $j$ th user for $x=0.5$ and considering perfect and imperfect simultaneous channel identifications, respectively. The results are shown for $M N K=4,7$, and 10, and with $L=2$ and $L=5$, respectively. Throughout the results, in the case when the cardinality of the set of interference-free channels $\mathcal{B}_{j}$ is less than a specific value $z$, where the $z$ th best available channels is identified, then the worst available channel is selected. From the results, it is clear that selecting a better channel from order statistics gives an improvement in performance per an active user. Hence, it is seen from both Figs. 4 and 5 that perfect simultaneous channel identification provides the best possible performance. However, the achieved performance reduces with the increase in the number of users due to the decrease in the likelihood of finding interferencefree channels, as discussed previously. Moreover, for the case of perfect channel identification, the performance improves with the increase in the number of available channels. The worst channel scenario where the worst channel is selected shows the opposite behaviour of the perfect best channel identification case. If the worst channel in the set of available channels is the one that is identified, then the performance improves with increase in number of users. This is because, as the expected number of interference-free channels decreases, the index of the worst channel increases. For example, if there only two interference-free channels, then the worst channel is the 2nd best channel and so on. This is also the reason why the performance of the worst channel case becomes better with decrease in the number of channels and the number of receive antennas in contrast with the best channel case. The behaviour of the second best channel identification and the third best channel identification cases hover between the perfect channel case and worst channel case. When there are less users, their behaviour mostly mirrors that of the perfect channel case. However, as the number of users increases, their performance starts to increase, on a small scale, due to the same reason as for the worst channel identification case.

The impact of imperfect user identification under perfect channel identification per each scheduled user is explained 


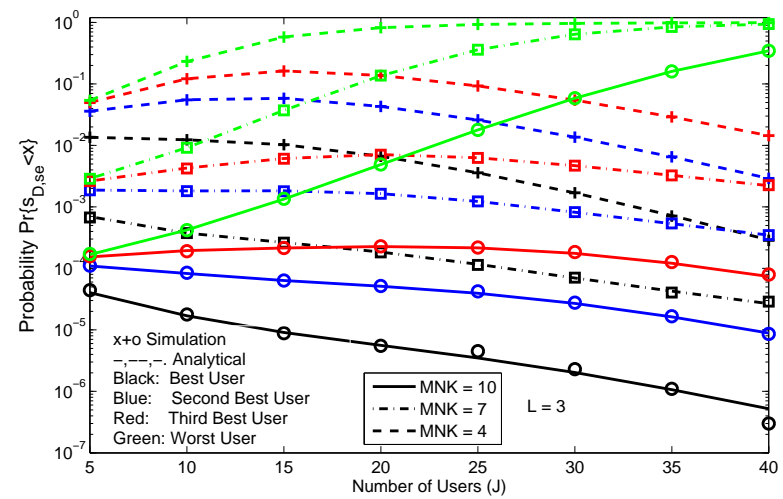

Fig. 7. Statistics of the scheduled user received desired power under perfect and imperfect scheduling, $\operatorname{Pr}\left\{s_{\mathrm{D}, \mathrm{se}}<x\right\}$ and $\operatorname{Pr}\left\{s_{\mathrm{D}, \widetilde{\mathrm{se}}}<x\right\}$ with $x=0.5$, and perfect channel identification per active user, as a function of the number of users $J=J_{0}$ with $M N K=4,7$, and 10 , and $L=3$.

in Figs. 6 and 7 for $L=2$ and $L=3$, respectively. The performance is characterized through $\operatorname{Pr}\left\{s_{\mathrm{D}, \mathrm{se}}<x\right\}$ and $\operatorname{Pr}\left\{s_{\mathrm{D}, \widetilde{\mathrm{se}}}<x\right\}$ with $x=0.5$. Here, it is considered that $J_{0}=J$. In this case, both figures lend some interesting observations. The probability of the scheduled user's channel being below a certain threshold is not a monotonic function of the number of scheduled users $J_{0}=J$, in general, even for the case of perfect user identification. The reason for this is that increasing the number of users has two conflicting outcomes. On one hand, it increases the chance of having an active user with relatively good downlink channel to access. On the other, it decreases the expected number of interference-free channels per each active user involved in multiuser scheduling. However, for a large number of users and an increased size of receive array per each user, the former outweighs the latter, particularly for the case of perfect user identification.

The case where imperfect identification occurs in both channel and scheduled user is depicted in Fig 8. The results are shown for the case of perfect best interference-free channel and best scheduled user identifications, best channel identification but second best user identification, best user identification but second best channel identification, and second best channel and second best user identifications. The obvious observation is that the performance results of the four scenarios above are bounded by the cases of perfect best identifications (lower bound) and imperfect second best identifications (upper bound). The results in between, i.e. one is perfect and the other is imperfect, offer some interesting insights. With a relatively small number of users, the results indicate that imperfectness in best interference-free channel identification (i.e., identifying the second best channel in order statistics) per active user with perfect best user scheduling degrades performance more than imperfectness in user identification with perfect best channel identification per each scheduled user, assuming that the imperfectness in channel identification is the same order for all active users involved in multiuser scheduling. This is due to the fact that the comparison between users performance results is done based on the second best user scheduling as compared to the best user scheduling. As the number of users increases,

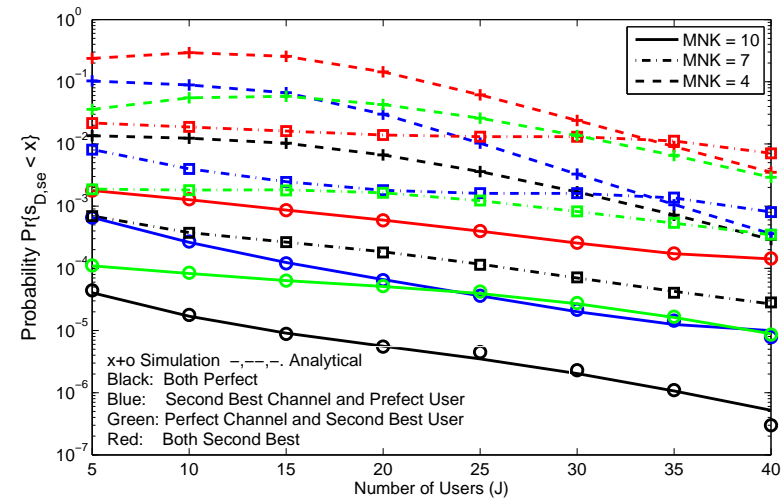

Fig. 8. Statistics of the scheduled user received desired power under perfect and imperfect scheduling, $\operatorname{Pr}\left\{s_{\mathrm{D} \text {, se }}<x\right\}$ and $\operatorname{Pr}\left\{s_{\mathrm{D}, \widetilde{\mathrm{se}}}<x\right\}$ with $x=0.5$, and considering perfect and imperfect channel identification per active user, as a function of the number of users $J=J_{0}$ with $M N K=4,7$, and 10, and $L=3$.

the two cases become closer in performance, and interestingly the case of perfect user identification with imperfect channel identification even outperforms its imperfect user identification and perfect channel counterpart due to the availability of an increased number of active users for multiuser scheduling. However, this is not universally true for increasing number of users as can be observed that the performance of the perfect channel identification and imperfect user identification case overtakes that of the imperfect channel identification and perfect user identification case. Hence, for a moderate number of users, the case of imperfect user identification with perfect channel identification provides better performance than the case of imperfect channel identification and perfect user identification. However, this is only true for a relatively large number of available channels. For a relatively small number of available channels, the latter outperforms the former.

\section{CONCLUSIONS}

This paper has investigated low-complexity approaches for joint interference avoidance and desired link improvement in multiuser multi-antenna open-access small cells. The adopted model has considered that an active user is equipped with highly-correlated receive antennas, and it can access only one downlink channel at a time. On the other hand, the APs operation can be coordinated to achieve the design and operation requirements, and each of which can unconditionally utilize available physical channels. Moreover, each AP is equipped with uncorrelated antennas that can be reused simultaneously to serve many active users. The analysis has provided new approaches to exploit the availability of physical channels, transmit antennas, and APs to mitigate interference, while providing the best possible link gain to an active user through the identification of the most suitable interference-free downlink channel. The event of concurrent service requests placed on a specific interference-free channel has been treated in the context of either interference avoidance through identifying unshared interference-free channels per active user or desired link improvement via multiuser scheduling. The usefulness of 
the proposed approaches to balance the distribution of downlink loads has been clarified. Moreover, practical scenarios due to imperfect identification of interference-free channel and/or imperfect identification of scheduled user have been treated for various scenarios. The developed results in this paper are generally applicable for any statistical and geometric channel models and conditions of interference users, and they can be used to study various performance measures. Numerical and simulation examples have also been presented, which showed that the chance of finding an interferencefree channel significantly increases even with relatively large number of users. This has decreased the number of search packet durations to find an interference-free channel per active user. Moreover, the noticeable effect of imperfect channel identification and/or imperfect scheduled user identification on the achieved performance has been thoroughly addressed.

\section{REFERENCES}

[1] H. Claussen, L. T. W. Ho, and L. G. Samuel, "An overview of the femtocell concept," Bell Labs Tech. Jour., vol. 13, no. 1, pp. 221-245, May 2008.

[2] V. Chandrasekhar, J. G. Andrews, and A. Gatherer, "Femtocell networks: a survey," IEEE Commun. Mag., vol. 46, no. 9, pp. 59-67, Sept. 2008.

[3] L. Balraj and R. Kavitha, "Deployment of femtocell and antenna in commercial building in cost effective way," in Proc. Fourth Int. Conf. Comput., Commun. Netw. Technol. (ICCCNT), 2013, pp. 1-5.

[4] J. Zhang, F. Jin, R. Zhang, G. Li, and L. Hanzo, "Distributed Antenna aided twin-layer femto-and macro-cell networks relying on fractional frequency-reuse," in Proc. IEEE Wireless Commun. Netw. Conf. (WCNC), 2013, pp. 1586-1591.

[5] R. M. Radaydeh and M.-S. Alouini, "Low-overhead interference mitigation scheme for collaborative channel assignment in overloaded multiantenna femtocells," IEEE Trans. Veh. Technol., vol. 61, no. 7, pp. 30713086, Jul. 2012.

[6] Z. Liu, J. Wang, Y. Xia, and H. Yang, "Robust optimisation of power control for femtocell networks," IET Signal Process., vol. 7, no. 5, pp. 360-367, Jul. 2013.

[7] A Dudnikova and D. Panno, "A fully distributed algorithm for pilot power control in LTE femtocell networks," in Proc. IFIP Wireless Days (WD), 2013, pp. 1-3.

[8] V. Chandrasekhar, J. G. Andrews, T. Muharemovic, Z. Shen, and A. Gatherer, "Power control in two-tier femtocell networks," IEEE Trans. Wireless Commun., vol. 8, no. 8, pp. 4316-4328, Aug. 2009.

[9] S. E. Nai, T. Quek, and M. Debbah, "Shadowing time-scale admission and power control for small cell networks," in Proc. 15th Int. Symp. Wireless Person. Multimedia Commun. (WPMC), 2012, pp. 628-632.

[10] D. Xenakis, N. Passas, L. Merakos, and C. Verikoukis, "Mobility management for femtocells in LTE-advanced: key aspects and survey of handover decision algorithms," IEEE Commun. Surveys \& Tut., vol. 16, no. 1, pp. 64-91, Feb. 2014.

[11] J. Gambini and U. Spagnolini, "Wireless over cable for femtocell systems," IEEE Commun. Mag., vol. 51, no. 5, pp. 178-185, May 2013.

[12] H. ElSawy, E. Hossain, and D. I. Kim, "HetNets with cognitive small cells: user offloading and distributed channel access techniques," IEEE Commun. Mag., vol. 51, no. 6, pp. 28-36, Jun. 2013.

[13] S. Sardellitti and S. Barbarossa, "Distributed RLS estimation for cooperative sensing in small cell networks," in Proc. IEEE Int. Conf. Acoustics, Speech and Signal Process. (ICASSP), 2013, pp. 5283-5287.

[14] R. M. Radaydeh, K. A. Qaraqe, and M.-S. Alouini, "Reducedcomplexity adaptive multi-channel assignment for shared access points in over-loaded small-cell networks," in Proc. IEEE Veh. Technol. Conf. (VTC Spring), 2013, pp. 1-5.

[15] R. M. Radaydeh, M.-S. Alouini, and K. A. Qaraqe, "Adaptive interference-aware multichannel assignment for shared overloaded smallcell access points under limited feedback," IEEE Trans. Veh Technol., vol. 63, no. 2, pp. 747-762, Feb. 2014.

[16] W. Ni, R. P. Liu, I. B. Collings, and X. Wang, "Indoor cooperative small cells over ethernet," IEEE Commun. Mag., vol. 51, no. 9, pp. 100-107, Sept. 2013.
[17] R. M. Radaydeh and M.-S. Alouini, "Switched-based interference reduction scheme for open-access overlaid cellular networks," IEEE Trans. Wireless Commun., vol. 11, no. 6, pp. 2160-2172, Jun. 2012.

[18] R. M. Radaydeh and M.-S. Alouini, "Low-complexity co-tier interference reduction scheme in open-access overlaid cellular networks," in Proc. IEEE Global Telecommun. Conf. (GLOBECOM), 2011, pp. 1-6.

[19] F. Gaaloul, R. M. Radaydeh, and M.-S. Alouini, "Performance improvement of switched-based interference mitigation for channel sssignment in over-loaded small-cell networks," IEEE Trans. Wireless Commun., vol. 12, no. 5, pp. 2091-2103, May 2013.

[20] G. Gür, S. Bayhan, and F. Alagöz, "Cognitive femtocell networks: an overlay architecture for localized dynamic spectrum access," IEEE Wireless Commun., vol. 17, no. 4, pp. 62-70, Aug. 2010.

[21] W. Wang, G. Yu, and A. Huang, "Cognitive radio enhanced interference coordination for femtocell networks," IEEE Commun. Mag., vol. 51, no. 6, pp. 37-43, Jun. 2013.

[22] A. Golaup, M. Mustapha, and L. B. Patanapongpibul, "Femtocell access control strategy in UMTS and LTE," IEEE Commun. Mag., vol. 47, no. 9, pp. 117-123, Sept. 2009.

[23] G. de la Roche, A. Valcarce, D. López-Pérez, and J. Zhang, "Access control mechanisms for femtocells," IEEE Commun. Mag., vol. 48, no. 1, pp. 33-39, Jan. 2010.

[24] A. Magableh, R. M. Radaydeh, and M.-S. Alouini, "On the performance of shared access control strategy for femtocells," Trans. Emerging Telecommun. Technol., vol. 24, no. 2, pp. 244-256, Mar. 2013.

[25] M. Rumney, LTE and the Evolution to $4 G$ Wireless: Design and Measurement Challenges, 2nd ed., Agilent Technologies, 2013.

[26] D. W. Kifle, B. Wegmann, P. Eskelinen, I. Viering, and A. Klein, "Super-cell from inner sectors of active antenna system (AAS) - vertical sectorization," in Proc. IEEE Int. Conf. Commun. (ICC), 2014, pp. 50955100 .

[27] Nokia Corp., "Indoor deployment strategies", Nokia White paper (Product code C401-00991-WP-201405-1-EN), Jun. 2014.

[28] S. V. Hum and J. Perruisseau-Carrier, "Reconfigurable reflectarrays and array lenses for dynamic antenna beam control: a review," IEEE Trans. Ant. Propag., vol. 62, no. 1, pp. 183-198, Jan. 2014.

[29] H. Steyskal, "Simple method for pattern nulling by phase perturbation," IEEE Trans. Ant. Propag., vol. 31, no. 1, pp. 163-166, Jan. 1983.

[30] W.-P. Liao and F.-L. Chu, "Array pattern nulling by phase and position perturbations with the use of the genetic algorithm," Microw. Optic. Technol. Lett., vol. 15, no. 4, pp. 251-256, Jul. 1997.

[31] R. Vescovo, "Null synthesis by phase control for antenna array," Electron. Lett., vol. 36, no. 3, pp. 198-199, Feb. 2000.

[32] Y. H. Wang, "On the number of successes in independent trials," Statistica Sinica, vol. 3, pp. 295-312, 1993.

[33] L. Le Cam, "An Approximation Theorem for the Poisson Binomial Distribution," Pacific Jour. Math., vol. 10, pp. 1181-1197, 1960.

[34] R. M. Radaydeh and M.-S. Alouini, "Comparisons of receive array interference reduction techniques under erroneous generalized transmit beamforming," IEEE Trans. Commun., vol. 62, no. 2, pp. 600-615, Feb. 2014.

[35] H. A. David and H. N. Nagaraja, Order Statistics, 3rd ed., Wiley, New York, 2003. 


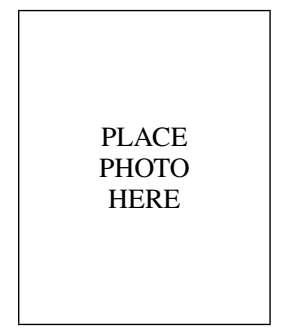

Redha M. Radaydeh (S'05, M'07, SM'13) was born in Irbid, Jordan, on November 12, 1978. He received the B.S. and M.S. degrees from Jordan University of Science and Technology (JUST), Irbid, in 2001 and 2003, respectively, and the Ph.D. degree from The University of Mississippi, Oxford, MS, USA, in 2006, all in Electrical Engineering. From February 2007 to September 2009, he was an Assistant Professor with the Department of Electrical Engineering, JUST. From October 2009 to January 2012, he was a Research Fellow with the Division of Physical Sciences and Engineering, King Abdullah University of Science and Technology (KAUST), Thuwal, Saudi Arabia. From January 2012 to September 2012, he was an Associate Research Scientist with the Electrical and Computer Engineering Program, Texas A\&M University at Qatar, Doha, Qatar. In September 2012, he joined Alfaisal University, Riyadh, Saudi Arabia, where he is currently working as an Associate Professor of Electrical Engineering. His research interests include wireless communication theory, energy-efficient communications, and design and performance analysis of wireless networks.

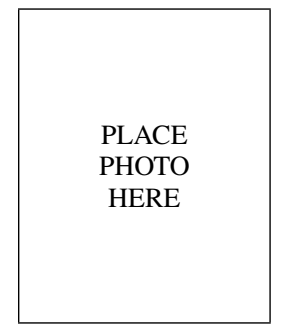

Ammar Zafar obtained his PhD in Wireless Communications from King Abdullah University of Science and Technology under the supervision of Dr. Mohamed-Slim Alouini in 2014. In November 2014, he joined Centre of Real-time Information Networks at the University of Technology Sydney as a postdoctoral research fellow working primarily with Prof. Guoqiang Mao. Ammar Zafar's research interests include traffic flow monitoring, vehicular networks, multi-user scheduling and wireless communication theory.

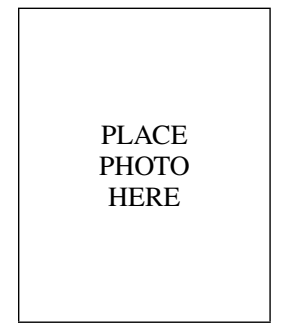

Fawaz S. Al-Qahtani (M'10) received the B.Sc. degree in electrical engineering from King Fahad University of Petroleum and Minerals, Dhahran, Saudi Arabia, in 2000, the M.Sc. degree in digital communication systems from Monash University, Melbourne, Australia, in 2005, and the Ph.D. degree in electrical and computer engineering from RMIT University, Melbourne, Australia, in December 2009. He is currently an Assistant Research Scientist with the Department of Electrical and Computer Engineering, Texas A\&M University at Qatar, Doha, Qatar. His current research interests include channel modeling, applied signal processing, MIMO communication systems, cooperative communications, cognitive radio systems, free-space optical, physical-layer security, visible light communication, device-to-device communication, and power transfer. Dr. Al-Qahtani was the recipient of sponsorship from the Qatar National Research Fund as well as from JSERP and NPRP projects. $\mathrm{He}$ is the author or coauthor of 70 over papers in refereed mainstream journals and reputed international conferences. He was the recipient of the research excellence award from Texas A\&M University at Qatar in 2013.

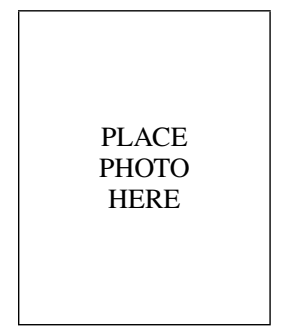

Mohamed-Slim Alouini (S'94, M'98, SM'03, F'09) was born in Tunis, Tunisia. He received the Ph.D. degree in Electrical Engineering from the California Institute of Technology (Caltech), Pasadena, CA, USA, in 1998. He served as a faculty member in the University of Minnesota, Minneapolis, MN, USA, then in the Texas A\&M University at Qatar, Education City, Doha, Qatar before joining King Abdullah University of Science and Technology (KAUST), Thuwal, Makkah Province, Saudi Arabia as a Professor of Electrical Engineering in 2009. His current research interests include the modeling, design, and performance analysis of wireless communication systems. 